The data obtained on leather are indicative of a compressibility that is intermediate between the compressibilities exhibited by most solids and those of liquids. The compression is not materially affected by moisture content. Values obtained for chrome-tanned leather and two types of collagen are in good agreement, but the compressions of vegetable and retanned leathers appear to be slightly different. Compressions of samples taken from different areas of the hide are in substantial agreement.

This investigation was made possible by the wholehearted cooperation of members of the Geophysical Laboratory of the Carnegie Institution of Washington and of the Bureau of Standards. The author is particularly indebted to L. H. Adams, H. S. Yoder, and J. Van den Huerk, of the Geophysical Laboratory, for encouragement, invaluable technical advice, and use of the facilities of their laboratory; and to E. F. Mueller and R. E. Wilson, of this Bureau, for advice and assistance in connection with the resistance measurements.

\section{References}

[1] C. E. Weir, J. Research NBS 41, 279 (1948) RP1924.

[2] C. E. Weir, J. Research NBS 42, 17 (1949) RP1947.

[3] C. E. Weir, J. Research NBS 44, 599 (1950) RP2106.

[4] C. E. Weir, J. Research NBS 35, 257 (1945) RP1672.

[5] L. H. Adams, E. D. Williamson, and J. Johnston, J. Am. Chem. Soc. 41, 12 (1919).

[6] P. W. Bridgman, Pro. Am. Acad. Arts \& Sci. 49, 627 (1914).

[7] L. H. Adams, R. W. Goranson, and R. E. Gibson, Rev. Sci. Instr. 8, 230 (1937)

[8] P. W. Bridgman, Pro. Am. Acad. Arts \& Sci. 47, 441 (1911).

[9] P. W. Bridgman, Pro. Am. Acad. Arts \& Sci. 47, 321 (1911).

[10] P. W. Bridgman, Pro. Am. Acad. Arts \& Sci. 74, 11 (1940).

[11] S. Timoshenko, Theory of elasticity (McGraw Hill Book Co., Inc., New York, N. Y. 1934)

[12] J. R. Kanagy, J. Am. Leather Chem. Assoc. 36, 609 (1941).

[13] L. H. Adams, J. Am. Chem. Soc. 53, 3769 (1931).

Washington, August 3, 1950.

\title{
Arc and Spark Spectra of Technetium
}

\author{
By William F. Meggers and Bourdon F. Scribner
}

\begin{abstract}
Four milligrams of highly purified technetium, loaned by the United States Atomic Energy Commission, were used to obtain a description of the arc and spark spectra characteristic of this fission product. Solutions containing 50 to 200 micrograms of technetium were dried on copper electrodes and excited by electric arcs or sparks. A stigmatic concave grating of 22-feet radius was employed to photograph the spectra from 2200 to 9000 angstroms, within which limits more than 2,300 lines characteristic of Tc atoms or ions were recorded. Wavelengths were measured relative to iron standards, relative intensities were estimated on a scale of 1 to 1000, and almost every line was definitely assigned either to neutral Tc atoms or to singly charged $\left(\mathrm{Tc}^{+}\right)$ions. The measured wavelengths range from 2261.30 to 8829.80 angstroms with average probable errors rarely exceeding \pm 0.01 angstrom. The average results of wavelength measurements and of intensity estimates for 2,121 lines in Te I and Te II spectra are presented. The strongest Te I lines have wavelengths $4297.06,4262.26,4238.19,4031.63$, and 3636.10 angstroms. The strongest Te II lines have wavelengths $2543.24,2610.00$, and 2647.02 angstroms. This description of Te I and Te II spectra will serve for spectro-chemical identification and for structural analyses of these spectra.
\end{abstract}

\section{Introduction}

In 1869, when the periodic chart of the atoms was first proposed, Mendeléeff $[1]^{1}$ saw that the chemical elements homologous to manganese were missing in the two succeeding periods; he called these ekamanganese and dwi-manganese. This was the first hint of the possible existence of the element under discussion. In the following half century many attempts, both chemical and spectroscopic, were made to detect these homologs of manganese, but without success. Moseley's [2] discovery in 1913 of a quantitative relationship between Roentgen frequencies and atomic numbers proved that atomic numbers $43,61,72,75,85$, and 87 were still unknown, and provided a new method of detection by means of Roentgen spectra. This method was applied in 1925 by Noddack, Tacke, and Berg [3], who reported

Figures in brackets indicate the literature references at the end of this paper. finding, in material extracted from columbite, three $K$ spectrum lines for atomic number 43 and five $L$ spectrum lines for atomic number 75 . These two atomic numbers are identical with the homologs of manganese, and the names masurium and rhenium were proposed for them. The discovery of rhenium was abundantly confirmed, and within a few years this new element became a commercial metal. In 1931 the arc spectrum of rhenium was described and interpreted by Meggers [4], who then inquired of the discoverers if a sample of masurium was available for spectroscopic investigation. The Noddacks ${ }^{2}$ replied that they had succeeded in concentrating some masurium, and that they would send the first milligram that could be spared. Unfortunately that milligram never materialized, and it later appeared that the extraction of element 43 from natural sources would be unlikely or even impossible [5].

\footnotetext{
${ }^{2}$ In a letter dated Dec. 22, 1931, from W. and I. Noddak to W. F. Meggers.
} 
After the discbvery of artificial radioactivity in 1934, it became theoretically possible to manufacture any desired element by artificial nuclear transmutation. Thus element 43 was first prepared in 1937 in the Berkeley cyclotron by bombarding molybdenum with neutrons or deuterons. It was identified, and its radiochemical properties were investigated by Perrier and Segré [6], who named the element technetium [7] because it was the first new element to be produced artificially. The amount of technetium produced in this manner was so small that it could only be detected by means of its characteristic radioactivity, which is enormously more sensitive than either roentgen or optical spectra. However, soon after the discovery of uranium fission in 1939, it was found that element 43 occurs among fission products to the extent of 6.2 percent [8]. In 1948 the isolation of milligram amounts of element 43 from uranium fission was described by Parker, Reed, and Ruch [9]. With these samples the whole-number atomic mass was determined by Inghram [10] to be 99, the first optical spectrogram was made by Timma [11], and the Roentgen emission spectrum was photographed by Burkhart, Peed, and Saunders [12]. Thus far the published information on the optical spectra of $\mathrm{Tc}$ consists of estimated relative intensities and approximate wavelengths of 102 lines ( 4297.2 to $2461.7 \mathrm{~A}$ ) attributed [11] to element 43. In the light of present information critical examination of those data discloses that possibly 46 of the 102 lines are identifiable as Ter, and 32 as TeIr, but the remaining 24 do not belong to Tc.

In September 1948, the National Bureau of Standards applied to the United States Atomic Energy Commission for the loan of $5 \mathrm{mg}$ of ${ }_{43}^{99} \mathrm{Tc}$ with which to make descriptions and analyses of the atomic and ionic emission spectra of technetium. A 3-mg sample was delivered in January 1949. With this sample preliminary results were obtained for wavelengths and relative intensities of about 2,000 Tc lines. These results were reported at the Oak Ridge Spectroscopy Symposium on March 25, 1949 [13] and at the Buffalo meeting of the Optical Society of America on October 29, 1949 [14]. In July 1950 three additional milligrams of ${ }_{43}^{99} \mathrm{Tc}$ were borrowed for the purpose of completing this description of arc and spark spectra and for the investigation of hyperfine structure of certain spectral lines. The purpose of this paper is to present the wavelengths and relative intensities of 2,121 lines that are characteristic of Tc atoms and ions. These data are suitable for spectrochemical identification and for structural analyses of the first two spectra of Tc. Details of the structural analyses of TcI and TeII spectra, and of the investigation of hyperfine structure in Tc spectral lines will be given in subsequent papers.

\section{Technetium Spectrograms}

The technetium samples came in the form of oxide. These samples were dissolved in dilute ammonium hydroxide to make solutions of known concentration, portions of which could then be added to spectro- scopic electrodes so that a definite amount of Tc was deposited on each. Fifty or one hundred micrograms of Te were dried on the flat-machined end of a copper $\operatorname{rod} 1 / 4 \mathrm{in}$. in diameter and 1 in. long. Electrodes with approximately rectangular ends, 6 by $2 \mathrm{~mm}$, were later employed to concentrate more of the light along the optical axis. Two such loaded electrodes were used when oscillatory discharges were employed for exciting the spectra, but when the direct-current are was employed the best results were obtained by placing $200 \mu \mathrm{g}$ on the anode. With a desire to economize material, the first spectrograms were made in the blue and ultraviolet with $50 \mu \mathrm{g}$ on each electrode, but to obtain stronger spectrograms it was necessary to use 100 on each. Also it was desirable to choose light sources that would burn the entire added sample off the ends of the copper electrodes without predominant excitation of the copper spectra. Furthermore, it was necessary to choose two sources with sufficiently different excitation characteristics so that comparisons of spectral line intensities would permit positive assignment of the lines either to neutral atoms or to ionized atoms. These considerations led to the choice of two sets of excitation conditions available in the Applied Research Laboratory Multisource unit.

The spark spectrum was obtained from an oscillatory condenser discharge with the following circuit constants: capacitance $5 \mu \mathrm{f}$, charging voltage $940 \mathrm{v}$, inductance $100 \mu \mathrm{h}$, and resistance $0.4 \mathrm{ohm}$. The arc spectrum was obtained from an overdamped condenser discharge with circuit constants: capacitance $50 \mu$ f, charging voltage $940 \mathrm{v}$, inductance $400 \mu \mathrm{h}$ and resistance 25 ohms. The gap between the electrodes was set at $4 \mathrm{~mm}$.

Before any Tc spectrograms were attempted, experiments were made with Mo spectrograms, and source conditions were determined that gave a fivefold enhancement of MoII lines over Mor lines when the spark spectrum was compared with the are. These source conditions described above were found to be suitable for an unambiguous separation of Ter and Teri lines.

It was immediately seen that the distribution of Te r and Te Ir lines resembled the distribution of first and second spectral lines of other metals; the second spectrum predominates in the ultraviolet and weakens in the visible, whereas the first spectrum is weak in the ultraviolet but predominates in longer waves. Because few Tc Ir lines are seen in the blue, the Tc spark spectrograms were not extended beyond $4700 \mathrm{~A}$.

Although the arc-like excitation provided by the ARL Multisource unit was satisfactory for the short wave region, it was disappointing for the visible and infrared, partly because it gave weak spectra and partly because it excited the spectra of atmospheric nitrogen and oxygen with such intensity that many portions of the Tc spectra were masked. Consequently, the spectral range 4500 to $9000 \mathrm{~A}$ was reobserved with a direct-current are between $1 / 8$-in. diameter $\mathrm{Cu}$ electrodes, the anode being charged with $200 \mu \mathrm{g}$ of Tc. These direct-current arc spectro- 
grams were made with an applied potential of $220 \mathrm{v}$ and a current of 7 amps.

The spectrograms were made with a concave grating of $22-\mathrm{ft}$ radius, ruled with 15,000 lines per inch on an aluminized pyrex disk of 6 -in. diameter. This grating was placed in a Jarrell-Ash instrument provided with a concave mirror of similar form, so that the spectrograph gave stigmatic slit images. A fixed slit of $20-\mu$ width was used, and the maximum resolving power for sharp lines in the first order spectrum was about 50,000, the reciprocal linear dispersion being about $5 \mathrm{~A} / \mathrm{mm}$.

Three positions of the grating and 20-in. plate holder recorded the spectrum between 2100 and 9000 A with successive exposures on three types of photographic plates. The region 2200 to $4700 \mathrm{~A}$ was photographed on Eastman 103-uv sensitized plates, the region 4400 to $6900 \mathrm{~A}$ on II-F sensitized plates, and 6500 to $9000 \mathrm{~A}$ on $\mathrm{I}-\mathrm{N}$ hypersensitized plates.

By sliding a slotted diaphragm in front of the slit, a series of adjacent spectrograms were made as follows: Iron arc, copper arc, technetium arc on copper, technetium spark on copper, copper spark, iron arc. Spark spectra were omitted in the visible and near infrared regions. The exposure durations were usually 1 sec for the iron arc, 45 sec for the copper blanks, and 30 sec for copper with technetium added. Preliminary tests with Mo added to copper showed that the sample was almost completely burned off in 30 sec. All exposures with $\mathrm{Tc}$ deposited on $\mathrm{Cu}$ were made with the electrodes mounted in a cylindrical brass cell provided with a quartz window and with an air exhaust through a glass-wool filter designed to trap, and thus conserve, most of the vaporized Tc for future recovery.
At least three satisfactory spêctrograms were made for each of the three spectral ranges mentioned. This gave at least six independent determinations for lines appearing in the overlapping portions of these ranges. The positions of Tc spectral lines relative to iron standards were measured to $0.001 \mathrm{~mm}$ with an excellent 50-cm comparator constructed by the Gaertner Scientific Corp. In all, approximately 20,000 spectral line images were bisected to obtain the wavelengths of about 2,500 Tc and impurity lines. All lines appearing in the spectra of $\mathrm{Cu}$ plus $\mathrm{Tc}$ that were not duplicated or masked in the $\mathrm{Cu}$ comparison spectra were measured, even though some were easily recognized as impurities. The final values of all the impurity lines served as checks on the scale of wavelengths and on the amazingly high purity of the Tc samples. The principal impurities detected spectroscopically were calcium, magnesium, and silicon, which are common contaminants in chemical operations. Only the stronger lines of lithium, sodium, and potassium were present, and the same is true of iron, nickel, chromium, molybdenum, aluminum, bismuth, and platinum. No trace of ruthenium, rhenium, or any other metals was found. In the identification and elimination of impurity lines, consideration was given to relative intensities as well as to wavelengths. Thus, the line 2496.77 A, with intensity 200 in Te II, cannot be confused with boron, 2496.778 A, because the stronger line of boron, $2497.733 \mathrm{~A}$, had only intensity 10 . A few doubtful coincidences of Tc and impurity lines are retained in table 1 , and it is not likely that many Tc lines have been masked by impurities. The chief omissions may be real Tc lines hidden behind the principal lines of copper, 3248, 3274, 5106, 5153, 5218, 5700, 5782, 7933, and $8093 \mathrm{~A}$.

TABLE 1. Arc and spark spectra of technetium

$\lambda=$ wavelength: $c=$ complex: $d=$ double: $h=$ hazy; $H=$ very hazy: $w=$ wide: $W=$ very wide: $s=$ shaded to shorter waves; $l=$ shaded to longer waves.

\begin{tabular}{|c|c|c|c|c|c|c|c|c|c|c|c|}
\hline \multirow{2}{*}{$\begin{array}{l}\lambda \\
A\end{array}$} & \multicolumn{2}{|c|}{ Intensity } & \multirow{2}{*}{$\begin{array}{l}\lambda \\
A\end{array}$} & \multicolumn{2}{|c|}{ Intensity } & \multirow{2}{*}{$\begin{array}{l}\lambda \\
A\end{array}$} & \multicolumn{2}{|c|}{ Intensity } & \multirow{2}{*}{$\begin{array}{l}\lambda \\
A\end{array}$} & \multicolumn{2}{|c|}{ Intensity } \\
\hline & Arc & Spark & & Arc & Spark & & Arc & Spark & & Arc & Spark \\
\hline $\begin{array}{l}8829.80 \\
8751.80 \\
8737.95 \\
8722.70 \\
8719.97\end{array}$ & $\begin{array}{l}10 c \\
1 \\
5 c W \\
2 \\
2\end{array}$ & & $\begin{array}{l}8308.14 \\
8254.48 \\
8248.95 \\
8237.08 \\
8228.65\end{array}$ & $\begin{array}{c}10 \\
2 \\
3 \\
15 c \\
1\end{array}$ & & $\begin{array}{l}\text { 7981. } 86 \\
7968.27 \\
7965.41 \\
7963.86 \\
7949.64\end{array}$ & $\begin{array}{l}1 \\
2 \\
5 \\
1 \\
2\end{array}$ & & $\begin{array}{l}7779.53 \\
7777.17 \\
7746.60 \\
7737.36 \\
7705.70\end{array}$ & $\begin{array}{l}1 \\
3 \\
2 \\
3 \\
1\end{array}$ & \\
\hline $\begin{array}{l}8716.77 \\
8707.21 \\
8652.76 \\
8543.63 \\
8537.66\end{array}$ & $\begin{array}{l}1 \\
9 \\
3 c \\
8 \\
2\end{array}$ & & $\begin{array}{l}\text { 8225. } 09 \\
8211.29 \\
8206.49 \\
8205.24 \\
8170.51\end{array}$ & $\begin{array}{c}3 c \\
10 \\
4 \\
10 \\
10\end{array}$ & & $\begin{array}{l}7885.96 \\
7874.72 \\
7871.16 \\
7863.11 \\
7861.36\end{array}$ & $\begin{array}{l}2 c \\
5 \\
50 \\
1 \\
30\end{array}$ & & $\begin{array}{l}7697.36 \\
7684.43 \\
7681.73 \\
7675.32 \\
7658.31\end{array}$ & $\begin{array}{c}80 c \\
8 \\
3 \\
1 \\
2\end{array}$ & \\
\hline $\begin{array}{l}8531.07 \\
8522.56 \\
8514.70 \\
8481.37 \\
8404.00\end{array}$ & $\begin{array}{l}7 \\
2 \\
2 \\
1 \\
1\end{array}$ & & $\begin{array}{l}8160.89 \\
8153.52 \\
8146.52 \\
8142.60 \\
8127.78\end{array}$ & $\begin{array}{l}2 \\
1 \\
1 \\
2 \\
1\end{array}$ & & $\begin{array}{l}7858.24 \\
7856.32 \\
7854.35 \\
7848.35 \\
7817.67\end{array}$ & $\begin{array}{r}2 \\
7 \\
1 \\
1 c \\
150 c\end{array}$ & & $\begin{array}{l}7624.49 \\
7618.39 \\
7579.20 \\
7573.99 \\
7550.98\end{array}$ & $\begin{array}{r}7 \\
3 \\
40 \\
15 \\
1\end{array}$ & \\
\hline $\begin{array}{l}8383.68 \\
8346.59 \\
8319.09 \\
8315.49 \\
8309.17\end{array}$ & $\begin{array}{l}3 \\
4 c \\
2 c \\
4 \\
10 c w\end{array}$ & & $\begin{array}{l}8119.87 \\
8068.80 \\
8045.30 \\
8042.45 \\
7999.68\end{array}$ & $\begin{array}{l}3 \\
2 c \\
2 \\
2 \\
10\end{array}$ & & $\begin{array}{l}\text { 7816. } 72 \\
7807.11 \\
7798.18 \\
7792.96 \\
7782.92\end{array}$ & $\begin{array}{c}5 \\
1 \\
4 \\
100 c W \\
2\end{array}$ & & $\begin{array}{l}7548.40 \\
7545.45 \\
7543.32 \\
7540.19 \\
7534.87\end{array}$ & $\begin{array}{r}4 \\
3 \\
7 \\
100 c \\
6\end{array}$ & \\
\hline
\end{tabular}


TABLE 1. Arc and spark spectra of technetium-Continued

\begin{tabular}{|c|c|c|c|c|c|c|c|c|c|c|c|}
\hline \multirow{2}{*}{$\begin{array}{l}\lambda \\
A\end{array}$} & \multicolumn{2}{|c|}{ Intensity } & \multirow{2}{*}{$\begin{array}{l}\lambda \\
A\end{array}$} & \multicolumn{2}{|c|}{ Intensity } & \multirow{2}{*}{$\begin{array}{l}\lambda \\
A\end{array}$} & \multicolumn{2}{|c|}{ Intensity } & \multirow{2}{*}{$\begin{array}{l}\lambda \\
A\end{array}$} & \multicolumn{2}{|c|}{ Intensity } \\
\hline & Arc & Spark & & Arc & Spark & & Are & Spark & & Arc & Spark \\
\hline $\begin{array}{l}7471.75 \\
7470.10 \\
7461.54 \\
7452.45 \\
7434.08\end{array}$ & $\begin{array}{l}1 c \\
5 \\
6 \\
50 c \\
10\end{array}$ & & $\begin{array}{l}6754.49 \\
6753.28 \\
6747.45 \\
6733.76 \\
6731.18\end{array}$ & $\begin{array}{l}1 c \\
1 \\
1 \\
1 \\
2 c w\end{array}$ & & $\begin{array}{l}6184.68 \\
6183.75 \\
6182.65 \\
6163.55 \\
6145.91\end{array}$ & $\begin{array}{l}5 \\
2 \\
3 \\
2 \\
1\end{array}$ & & $\begin{array}{l}\text { 5915. } 74 \\
5908.57 \\
5905.85 \\
5905.46 \\
5903.82\end{array}$ & $\begin{array}{l}1 \\
2 c \\
1 \\
2 \\
1\end{array}$ & \\
\hline $\begin{array}{l}7405.33 \\
7402.57 \\
7396.79 \\
7395.05 \\
7366.28\end{array}$ & $\begin{array}{l}15 \\
4 c d \\
8 c \\
4 c \\
2 c\end{array}$ & & $\begin{array}{l}6687.03 \\
6673.64 \\
6661.95 \\
6661.36 \\
6648.35\end{array}$ & $\begin{array}{l}3 \\
9 \\
1 \\
1 \\
2\end{array}$ & & $\begin{array}{l}6132.21 \\
6130.81 \\
6127.14 \\
6124.28 \\
6120.67\end{array}$ & $\begin{array}{r}8 c \\
60 \\
1 \\
1 \\
150\end{array}$ & & $\begin{array}{l}5893.57 \\
5885.37 \\
5879.26 \\
5874.74 \\
5873.60\end{array}$ & $\begin{array}{l}2 \\
2 c w \\
1 \\
2 \\
1\end{array}$ & \\
\hline $\begin{array}{l}7360.02 \\
7338.18 \\
7329.11 \\
7322.34 \\
7316.42\end{array}$ & $\begin{array}{c}1 \\
3 \\
8 c \\
10 c \\
1\end{array}$ & & $\begin{array}{l}6627.87 \\
6625.54 \\
6602.01 \\
6598.00 \\
6595.16\end{array}$ & $\begin{array}{l}3 \\
40 c W_{s} \\
1 \\
1 c \\
1 c\end{array}$ & & $\begin{array}{l}6115.44 \\
6111.98 \\
6111.06 \\
6102.95 \\
6099.38\end{array}$ & $\begin{array}{l}1 \\
2 \\
2 \\
60 c \\
20\end{array}$ & & $\begin{array}{l}\text { 5871. } 22 \\
5867.47 \\
5865.32 \\
5863.79 \\
5853.29\end{array}$ & $\begin{array}{l}2 \\
1 \\
1 \\
2 c \\
2 c\end{array}$ & \\
\hline $\begin{array}{l}7283.18 \\
7256.05 \\
7251.53 \\
7157.59 \\
7156.78\end{array}$ & $\begin{array}{c}1 c \\
4 \\
1 c \\
10 c \\
1\end{array}$ & & $\begin{array}{l}6589.96 \\
6579.23 \\
6576.79 \\
6563.99 \\
6534.57\end{array}$ & $\begin{array}{l}1 \\
9 \\
4 \\
3 \\
1\end{array}$ & & $\begin{array}{l}6093.71 \\
6085.22 \\
6069.77 \\
6066.15 \\
6065.08\end{array}$ & $\begin{array}{c}2 \\
100 c w s \\
4 \\
1 \\
20\end{array}$ & & $\begin{array}{l}5838.28 \\
5836.28 \\
5831.45 \\
5827.14 \\
5826.49\end{array}$ & $\begin{array}{c}1 \\
10 \\
30 \\
2 \\
4 c\end{array}$ & \\
\hline $\begin{array}{l}7141.23 \\
7093.08 \\
7086.11 \\
7071.38 \\
7048.49\end{array}$ & $\begin{array}{c}8 \\
4 c \\
20 c \\
3 \\
2 c\end{array}$ & & $\begin{array}{l}6533.98 \\
6526.79 \\
6515.11 \\
6496.58 \\
6491.65\end{array}$ & $\begin{array}{r}1 \\
15 \\
1 \\
1 c \\
15 c\end{array}$ & & $\begin{array}{l}6063.91 \\
6063.45 \\
6057.24 \\
6047.98 \\
6032.34\end{array}$ & $\begin{array}{l}3 c \\
4 \\
2 \\
4 \\
6\end{array}$ & & $\begin{array}{l}5823.09 \\
5821.24 \\
5819.11 \\
5814.20 \\
5805.19\end{array}$ & $\begin{array}{l}1 \\
1 \\
1 c \\
3 \\
2\end{array}$ & \\
\hline $\begin{array}{l}7029.51 \\
7019.95 \\
7016.50 \\
7006.22 \\
7002.33\end{array}$ & $\begin{array}{l}1 \\
1 \\
4 \\
1 c \\
7 c\end{array}$ & & $\begin{array}{l}6470.25 \\
6469.49 \\
6461.91 \\
6455.88 \\
6445.05\end{array}$ & $\begin{array}{r}7 \\
1 \\
40 \\
100 \\
3 c\end{array}$ & & $\begin{array}{l}6030.24 \\
6028.50 \\
6024.41 \\
6023.49 \\
6020.68\end{array}$ & $\begin{array}{l}1 \\
1 \\
2 \\
4 \\
1\end{array}$ & & $\begin{array}{l}5802.39 \\
5799.83 \\
5794.62 \\
5777.92 \\
5775.02\end{array}$ & $\begin{array}{l}1 \\
8 c \\
9 c \\
1 \\
1\end{array}$ & . \\
\hline $\begin{array}{l}6998.60 \\
6990.22 \\
6985.35 \\
6934.02 \\
6933.04\end{array}$ & $\begin{array}{l}1 \\
1 \\
1 \\
1 \\
1\end{array}$ & & $\begin{array}{l}6412.90 \\
6408.81 \\
6392.38 \\
6389.85 \\
6388.38\end{array}$ & $\begin{array}{l}1 c \\
5 \\
4 \\
6 \\
3 c\end{array}$ & & $\begin{array}{l}6018.59 \\
6013.17 \\
6012.07 \\
6010.17 \\
5997.12\end{array}$ & $\begin{array}{l}1 \\
1 \\
2 c \\
2 \\
1\end{array}$ & & $\begin{array}{l}5773.61 \\
5771.45 \\
5754.74 \\
5749.50 \\
5741.92\end{array}$ & $\begin{array}{l}2 \\
40 c w \\
2 \\
1 \\
1 c\end{array}$ & \\
\hline $\begin{array}{l}6931.72 \\
6907.50 \\
6876.69 \\
6874.59 \\
6871.65\end{array}$ & $\begin{array}{l}2 c \\
2 \\
2 c \\
1 \\
1\end{array}$ & & $\begin{array}{l}6356.71 \\
6354.83 \\
6312.15 \\
6310.40 \\
6306.16\end{array}$ & $\begin{array}{r}10 \\
15 \\
10 \\
4 \\
1\end{array}$ & & $\begin{array}{l}5996.67 \\
5993.19 \\
5986.76 \\
5980.57 \\
5974.79\end{array}$ & $\begin{array}{l}2 \\
1 c w \\
1 \\
4 c w \\
1\end{array}$ & & $\begin{array}{l}5737.52 \\
5725.32 \\
5721.08 \\
5719.52 \\
5696.61\end{array}$ & $\begin{array}{c}1 \\
40 \\
1 \\
1 \\
1 c\end{array}$ & \\
\hline $\begin{array}{l}6866.64 \\
6862.30 \\
6860.67 \\
6856.85 \\
6829.92\end{array}$ & $\begin{array}{l}1 \\
1 \\
2 \\
3 \\
1\end{array}$ & & $\begin{array}{l}6305.00 \\
6303.98 \\
6296.72 \\
6275.38 \\
6255.74\end{array}$ & $\begin{array}{l}3 \\
1 \\
1 c \\
1 \\
1\end{array}$ & & $\begin{array}{l}5968.98 \\
5951.46 \\
5946.48 \\
5942.24 \\
5938.38\end{array}$ & $\begin{array}{l}1 c \\
4 \\
1 \\
2 c w \\
3\end{array}$ & & $\begin{array}{l}\text { 5696. } 03 \\
5694.30 \\
5692.22 \\
5689.05 \\
5688.74\end{array}$ & $\begin{array}{c}2 \\
1 c \\
1 \\
15 \\
2\end{array}$ & \\
\hline $\begin{array}{l}6826.06 \\
6818.01 \\
6813.50 \\
6805.89 \\
6804.93\end{array}$ & $\begin{array}{l}1 \\
1 \\
1 \\
1 \\
1\end{array}$ & & $\begin{array}{l}\text { 6248. } 94 \\
6244.17 \\
6239.67 \\
6236.77 \\
6214.02\end{array}$ & $\begin{array}{c}2 \\
50 c \\
2 \\
1 c \\
3\end{array}$ & & $\begin{array}{l}\text { 5937. } 07 \\
5931.93 \\
5930.32 \\
5926.29 \\
\text { 5925. } 69\end{array}$ & $\begin{array}{r}1 \\
60 \\
2 \\
15 \\
2\end{array}$ & & $\begin{array}{l}5687.31 \\
5685.52 \\
5680.93 \\
5678.46 \\
5675.14\end{array}$ & $\begin{array}{r}15 \\
1 \\
1 \\
2 \\
1\end{array}$ & \\
\hline $\begin{array}{l}6798.58 \\
6785.95 \\
6776.50 \\
6761.40 \\
6758.26\end{array}$ & $\begin{array}{l}3 \\
3 \\
1 \\
2 \\
2\end{array}$ & - & $\begin{array}{l}\text { 6204. } 40 \\
6202.68 \\
6198.76 \\
6195.36 \\
6192.65\end{array}$ & $\begin{array}{c}1 \\
2 c w \\
1 \\
3 \\
100 c\end{array}$ & & $\begin{array}{l}5925.38 \\
5924.47 \\
5923.35 \\
5921.34 \\
5919.42\end{array}$ & $\begin{array}{c}3 c \\
100 c W \\
10 \\
1 \\
1\end{array}$ & & $\begin{array}{l}5672.15 \\
5667.59 \\
5667.03 \\
5664.89 \\
5662.09\end{array}$ & $\begin{array}{r}c \\
1 c\end{array}$ & \\
\hline
\end{tabular}


TABLE 1. Arc and spark spectra of technetium-Continued

\begin{tabular}{|c|c|c|c|c|c|c|c|c|c|c|c|}
\hline \multirow{2}{*}{$\begin{array}{l}\lambda \\
A\end{array}$} & \multicolumn{2}{|c|}{ Intensity } & \multirow{2}{*}{$\begin{array}{l}\lambda \\
A\end{array}$} & \multicolumn{2}{|c|}{ Intensity } & \multirow{2}{*}{$\begin{array}{l}\lambda \\
A\end{array}$} & \multicolumn{2}{|c|}{ Intensity } & \multirow{2}{*}{$\begin{array}{l}\lambda \\
A\end{array}$} & \multicolumn{2}{|c|}{ Intensity } \\
\hline & Arc & Spark & & Arc & Spark & & Are & Spark & & Arc & Spark \\
\hline $\begin{array}{l}5661.52 \\
5658.05 \\
5655.99 \\
5644.93 \\
5644.32\end{array}$ & $\begin{array}{r}2 \\
1 \\
5 \\
50 \\
1\end{array}$ & & $\begin{array}{l}5468.66 \\
5467.36 \\
5458.07 \\
5455.94 \\
5455.08\end{array}$ & $\begin{array}{l}1 \\
1 \\
1 c \\
3 \\
2 c\end{array}$ & & $\begin{array}{l}5240.92 \\
5236.10 \\
5231.22 \\
5230.28 \\
5228.67\end{array}$ & $\begin{array}{l}1 \\
1 \\
1 \\
1 \\
2 c\end{array}$ & & $\begin{array}{l}\text { 4980. } 41 \\
4978.52 \\
\text { 4976. } 34 \\
4967.18 \\
4956.32\end{array}$ & $\begin{array}{r}2 \\
1 \\
150 \\
2 \\
1\end{array}$ & \\
\hline $\begin{array}{l}5642.12 \\
5640.76 \\
5637.77 \\
5636.83 \\
5636.24\end{array}$ & $\begin{array}{c}100 \\
2 \\
1 \\
3 c \\
2\end{array}$ & & $\begin{array}{l}5451.89 \\
5450.41 \\
5448.35 \\
5447.76 \\
5447.39\end{array}$ & $\begin{array}{r}40 \\
1 \\
2 \\
1 \\
9\end{array}$ & & $\begin{array}{l}5227.53 \\
5214.08 \\
5208.52 \\
5206.53 \\
5202.10\end{array}$ & $\begin{array}{l}4 \\
1 \\
2 c \\
4 \\
1\end{array}$ & & $\begin{array}{l}\text { 4954. } 94 \\
4948.79 \\
\text { 4948. } 06 \\
4947.04 \\
4944.20\end{array}$ & $\begin{array}{l}1 \\
1 \\
8 \\
2 \\
1\end{array}$ & \\
\hline $\begin{array}{l}5629.92 \\
5625.20 \\
5620.45 \\
5617.63 \\
5616.76\end{array}$ & $\begin{array}{c}20 \\
1 \\
200 c \\
1 \\
1\end{array}$ & & $\begin{array}{l}5444.25 \\
5439.48 \\
5425.93 \\
5423.05 \\
5412.89\end{array}$ & $\begin{array}{l}2 \\
1 c \\
1 \\
5 \\
2\end{array}$ & & $\begin{array}{l}5193.10 \\
5189.67 \\
5184.95 \\
5183.97 \\
5178.05\end{array}$ & $\begin{array}{l}3 \\
2 \\
1 \\
2 \\
1\end{array}$ & & $\begin{array}{l}\text { 4938. } 79 \\
\text { 4936. } 10 \\
\text { 4933. } 79 \\
\text { 4929. } 71 \\
\text { 4923. } 58\end{array}$ & $\begin{array}{l}2 \\
1 \\
1 c \\
1 \\
4\end{array}$ & \\
\hline $\begin{array}{l}5615.23 \\
5611.46 \\
5606.37 \\
5603.52 \\
5602.22\end{array}$ & $\begin{array}{l}1 \\
2 \\
1 c \\
1 \\
10\end{array}$ & & $\begin{array}{l}5411.92 \\
5407.90 \\
5400.45 \\
5389.30 \\
5387.62\end{array}$ & $\begin{array}{l}2 \\
4 \\
1 \\
2 c \\
1\end{array}$ & & $\begin{array}{l}5177.69 \\
5174.79 \\
5167.75 \\
5161.77 \\
5150.58\end{array}$ & $\begin{array}{c}1 \\
100 \\
1 \\
80 c \\
10\end{array}$ & & $\begin{array}{l}\text { 4920. } 65 \\
4915.79 \\
4914.68 \\
4913.01 \\
4909.55\end{array}$ & $\begin{array}{r}3 \\
1 \\
2 \\
15 \\
100\end{array}$ & \\
\hline $\begin{array}{l}5596.53 \\
5589.01 \\
5584.00 \\
5580.19 \\
5577.57\end{array}$ & $\begin{array}{c}1 c \\
300 c d \\
2 \\
1 c \\
1 c\end{array}$ & & $\begin{array}{l}5386.86 \\
5383.45 \\
5382.64 \\
5377.39 \\
5376.27\end{array}$ & $\begin{array}{l}1 \\
1 c \\
1 \\
1 \\
1\end{array}$ & & $\begin{array}{l}5139.25 \\
5136.12 \\
5135.78 \\
5134.28 \\
5122.14\end{array}$ & $\begin{array}{c}10 \\
2 \\
1 \\
2 c \\
1\end{array}$ & & $\begin{array}{l}4908.50 \\
4906.39 \\
4900.01 \\
4892.47 \\
4891.89\end{array}$ & $\begin{array}{r}40 \\
1 \\
1 \\
3 \\
150\end{array}$ & \\
\hline $\begin{array}{l}5576.77 \\
5576.25 \\
5575.41 \\
5572.85 \\
5570.74\end{array}$ & $\begin{array}{l}2 \\
2 \\
1 c \\
2 \\
1 c\end{array}$ & & $\begin{array}{l}\text { 5375. } 19 \\
5366.02 \\
5364.01 \\
5360.65 \\
5359.21\end{array}$ & $\begin{array}{c}30 c \\
1 \\
1 \\
1 \\
2\end{array}$ & & $\begin{array}{l}5120.59 \\
5113.64 \\
5109.79 \\
5104.33 \\
5103.23\end{array}$ & $\begin{array}{c}3 \\
2 \\
8 \\
30 \\
10 c\end{array}$ & & $\begin{array}{l}4890.89 \\
4889.41 \\
4888.69 \\
4885.87\end{array}$ & $\begin{array}{l}3 \\
1 \\
2 \\
1\end{array}$ & \\
\hline $\begin{array}{l}5568.62 \\
5562.93 \\
5550.51 \\
5545.79 \\
5543.60\end{array}$ & $\begin{array}{l}1 c \\
1 \\
3 \\
1 c \\
2\end{array}$ & & $\begin{array}{l}5358.63 \\
5356.63 \\
5353.48 \\
5350.85 \\
5334.79\end{array}$ & $\begin{array}{r}15 \\
6 \\
50 \\
1 \\
15\end{array}$ & & $\begin{array}{l}5102.51 \\
5100.69 \\
5099.97 \\
5096.27 \\
5090.74\end{array}$ & $\begin{array}{r}2 \\
1 \\
1 \\
200 \\
3\end{array}$ & & $\begin{array}{l}4885.00 \\
4876.96 \\
4874.76 \\
4870.76 \\
4866.71\end{array}$ & $\begin{array}{c}1 \\
2 d \\
1 \\
3 \\
250\end{array}$ & \\
\hline $\begin{array}{l}5541.92 \\
5540.55 \\
5538.84 \\
5528.22 \\
5524.08\end{array}$ & $\begin{array}{l}7 \\
1 \\
2 c \\
4 \\
6\end{array}$ & & $\begin{array}{l}\text { 5328. } 42 \\
5320.20 \\
5318.16 \\
5317.51 \\
5314.97\end{array}$ & $\begin{array}{r}1 \\
30 \\
1 \\
2 \\
10\end{array}$ & & $\begin{array}{l}5086.91 \\
5083.94 \\
5069.54 \\
5060.69 \\
5058.32\end{array}$ & $\begin{array}{r}3 \\
2 \\
2 \\
30 \\
2\end{array}$ & & $\begin{array}{l}4863.29 \\
4862.91 \\
4862.19 \\
4861.19 \\
4859.19\end{array}$ & $\begin{array}{l}1 \\
1 \\
2 \\
1 \\
1\end{array}$ & \\
\hline $\begin{array}{l}5518.02 \\
5514.16 \\
5513.58 \\
5510.81 \\
5506.85\end{array}$ & $\begin{array}{l}2 \\
1 c \\
1 \\
1 \\
9 c w\end{array}$ & & $\begin{array}{l}\text { 5311. } 13 \\
5305.31 \\
\text { 5285. } 06 \\
\text { 5279. } 31 \\
\text { 5275. } 51\end{array}$ & $\begin{array}{r}1 \\
4 \\
30 \\
1 \\
50\end{array}$ & & $\begin{array}{l}5055.27 \\
5051.27 \\
5032.44 \\
5027.88 \\
5026.79\end{array}$ & $\begin{array}{l}9 \\
2 \\
3 \\
3 \\
5\end{array}$ & & $\begin{array}{l}4857.19 \\
4853.57 \\
4841.36 \\
4835.40 \\
4834.36\end{array}$ & $\begin{array}{r}2 \\
400 \\
2 \\
30 \\
30\end{array}$ & \\
\hline $\begin{array}{l}5505.25 \\
5502.93 \\
5500.04 \\
5495.96 \\
5490.32\end{array}$ & $\begin{array}{l}2 \\
2 \\
2 \\
1 \\
2\end{array}$ & . & $\begin{array}{l}\text { 5274. } 82 \\
\text { 5273. } 91 \\
\text { 5273. } 20 \\
\text { 5267. } 21 \\
\text { 5266. } 30\end{array}$ & $\begin{array}{l}2 \\
2 \\
1 \\
1 \\
1 c\end{array}$ & & $\begin{array}{l}5026.23 \\
5024.68 \\
5014.51 \\
5010.72 \\
5005.73\end{array}$ & $\begin{array}{r}15 \\
1 \\
4 \\
1 \\
2\end{array}$ & & $\begin{array}{l}\text { 4831. } 33 \\
4820.75 \\
4816.79 \\
4813.26 \\
4811.79\end{array}$ & $\begin{array}{r}3 \\
150 \\
9 \\
1 \\
1\end{array}$ & \\
\hline $\begin{array}{l}5485.32 \\
5484.99 \\
5482.97 \\
5482.83 \\
5471.92\end{array}$ & $\begin{array}{r}2 \\
2 \\
5 \\
3 \\
10\end{array}$ & & $\begin{array}{l}\text { 5261. } 44 \\
5254.74 \\
5251.39 \\
5249.37 \\
5246.97\end{array}$ & $\begin{array}{l}6 c \\
2 c \\
2 \\
1 \\
2\end{array}$ & & $\begin{array}{l}5002.67 \\
5000.82 \\
4999.07 \\
4995.00 \\
4993.97\end{array}$ & $\begin{array}{r}3 \\
1 \\
1 \\
15 \\
1\end{array}$ & & $\begin{array}{l}4809.37 \\
4805.63 \\
4800.62 \\
4799.96 \\
4791.62\end{array}$ & $\begin{array}{l}1 \\
1 \\
1 \\
3 \\
4\end{array}$ & \\
\hline
\end{tabular}


TABLE 1. Arc and spark spectra of technetium-Continued

\begin{tabular}{|c|c|c|c|c|c|c|c|c|c|c|c|}
\hline \multirow{2}{*}{$\begin{array}{l}\lambda \\
A\end{array}$} & \multicolumn{2}{|c|}{ Intensity } & \multirow{2}{*}{$\begin{array}{l}\lambda \\
A\end{array}$} & \multicolumn{2}{|c|}{ Intensity } & \multirow{2}{*}{$\begin{array}{l}\lambda \\
A\end{array}$} & \multicolumn{2}{|c|}{ Intensity } & \multirow{2}{*}{$\begin{array}{l}\lambda \\
A\end{array}$} & \multicolumn{2}{|c|}{ Intensity } \\
\hline & Arc & Spark & & Are & Spark & & Arc & Spark & & Arc & Spark \\
\hline $\begin{array}{l}4790.47 \\
4789.48 \\
4787.69 \\
4785.59 \\
4784.69\end{array}$ & $\begin{array}{l}3 \\
2 \\
2 c \\
4 \\
1\end{array}$ & & $\begin{array}{l}4616.86 \\
4609.17 \\
4608.00 \\
4602.72 \\
4594.06\end{array}$ & $\begin{array}{l}9 \\
4 c \\
2 \\
2 \\
2\end{array}$ & $\begin{array}{l}5 \\
3 h \\
1 \\
1 \\
1\end{array}$ & $\begin{array}{l}\text { 4328. } 77 \\
\text { 4324. } 85 \\
\text { 4323. } 94 \\
4323.35 \\
\text { 4319. } 94\end{array}$ & $\begin{array}{l}3 \\
1 \\
1 \\
3 \\
1 h\end{array}$ & 2 & $\begin{array}{l}\text { 4146. } 21 \\
4145.02 \\
4141.28 \\
4139.85 \\
4139.10\end{array}$ & $\begin{array}{c}1 \\
80 c \\
3 \\
5 \\
3\end{array}$ & $\begin{array}{c}50 c \\
2 \\
4 \\
2\end{array}$ \\
\hline $\begin{array}{l}4783.92 \\
4780.56 \\
4779.84 \\
4776.33 \\
4773.86\end{array}$ & $\begin{array}{l}2 \\
1 \\
1 \\
2 \\
2\end{array}$ & & $\begin{array}{l}4593.36 \\
4584.86 \\
4579.09 \\
4578.46 \\
4570.85\end{array}$ & $\begin{array}{r}20 \\
2 \\
1 \\
10 \\
2\end{array}$ & $\begin{array}{r}10 \\
1 \\
\\
6 \\
1\end{array}$ & $\begin{array}{l}\text { 4318. } 65 \\
\text { 4314. } 03 \\
4312.48 \\
4310.21 \\
4308.59\end{array}$ & $\begin{array}{l}2 \\
1 \\
1 \\
3 \\
2\end{array}$ & $\begin{array}{l}2 \\
1\end{array}$ & $\begin{array}{l}\text { 4134. } 80 \\
\text { 4130. } 44 \\
\text { 4128. } 27 \\
\text { 4124. } 22 \\
\text { 4119. } 28\end{array}$ & $\begin{array}{r}3 \\
2 \\
10 \\
80 \\
4\end{array}$ & $\begin{array}{r}2 \\
1 \\
6 \\
50 \\
3\end{array}$ \\
\hline $\begin{array}{l}4771.53 \\
4762.35 \\
4758.06 \\
4756.09 \\
4752.71\end{array}$ & $\begin{array}{r}150 \\
2 \\
1 \\
1 \\
4\end{array}$ & & $\begin{array}{l}4564.57 \\
4563.80 \\
4562.94 \\
4558.73 \\
4557.06\end{array}$ & $\begin{array}{r}30 \\
1 \\
1 \\
1 \\
6\end{array}$ & $\begin{array}{r}20 \\
1\end{array}$ & $\begin{array}{l}\text { 4307. } 78 \\
\text { 4305. } 82 \\
\text { 4305. } 52 \\
\text { 4297. } 06 \\
\text { 4294. } 35\end{array}$ & $\begin{array}{r}1 \\
4 \\
1 \\
500 \\
5\end{array}$ & $\begin{array}{r}1 \\
3 \\
300 \\
3\end{array}$ & $\begin{array}{l}\text { 4115. } 08 \\
\text { 4113. } 26 \\
4111.37 \\
4110.21 \\
4106.69\end{array}$ & $\begin{array}{r}100 \\
1 \\
2 \\
10 \\
2\end{array}$ & $\begin{array}{r}60 \\
1 \\
6 \\
1\end{array}$ \\
\hline $\begin{array}{l}4749.60 \\
4746.02 \\
4741.24 \\
4740.59 \\
4739.53\end{array}$ & $\begin{array}{r}3 \\
1 \\
1 \\
200 \\
1\end{array}$ & & $\begin{array}{l}4552.85 \\
4552.41 \\
4552.21 \\
4545.56 \\
4542.10\end{array}$ & $\begin{array}{l}5 \\
1 \\
3 \\
1 \\
4\end{array}$ & $\begin{array}{l}4 \\
2 \\
3\end{array}$ & $\begin{array}{l}4289.54 \\
4286.31 \\
4278.91 \\
4277.53 \\
4274.95\end{array}$ & $\begin{array}{c}2 \\
1 \\
40 \\
1 \\
3 ?\end{array}$ & $\begin{array}{c}1 \\
20 \\
2 ?\end{array}$ & $\begin{array}{l}\text { 4106. } 21 \\
\text { 4105. } 08 \\
\text { 4101. } 24 \\
\text { 4100. } 08 \\
\text { 4098. } 77\end{array}$ & $\begin{array}{l}1 \\
2 \\
2 \\
2 \\
1\end{array}$ & $\begin{array}{l}1 \\
1 \\
1\end{array}$ \\
\hline $\begin{array}{l}4736.48 \\
4719.28 \\
4717.76 \\
4716.76 \\
4714.20\end{array}$ & $\begin{array}{c}2 c \\
100 \\
10 \\
1 \\
2\end{array}$ & & $\begin{array}{l}4539.54 \\
4537.36 \\
4522.85 \\
4515.98 \\
4501.74\end{array}$ & $\begin{array}{r}100 \\
2 \\
200 \\
10 \\
1\end{array}$ & $\begin{array}{r}50 \\
1 \\
100 \\
6\end{array}$ & $\begin{array}{l}\text { 4266. } 16 \\
\text { 4262. } 67 \\
\text { 4262. } 26 \\
\text { 4258. } 61 \\
\text { 4257. } 33\end{array}$ & $\begin{array}{r}1 \\
100 \\
400 \\
1 \\
1\end{array}$ & $\begin{array}{r}1 \\
50 \\
200\end{array}$ & $\begin{array}{l}\text { 4095. } 68 \\
\text { 4094. } 17 \\
\text { 4093. } 68 \\
\text { 4091. 54 } \\
\text { 4090. } 13\end{array}$ & $\begin{array}{r}200 \\
2 \\
3 \\
2 \\
2\end{array}$ & $\begin{array}{r}100 \\
1 \\
2 \\
1 \\
1\end{array}$ \\
\hline $\begin{array}{l}4706.90 \\
4702.83 \\
4694.28 \\
4689.77 \\
4689.34\end{array}$ & $\begin{array}{l}6 \\
1 \\
3 \\
1 \\
4\end{array}$ & & $\begin{array}{l}4498.54 \\
4495.03 \\
4487.05 \\
4481.52 \\
4473.65\end{array}$ & $\begin{array}{l}1 h \\
10 \\
40 \\
10 \\
1\end{array}$ & $\begin{array}{r}5 \\
20 \\
5\end{array}$ & $\begin{array}{l}4243.00 \\
4239.55 \\
4238.19 \\
4232.09 \\
4230.84\end{array}$ & $\begin{array}{r}2 \\
2 \\
300 \\
2 \\
6\end{array}$ & $\begin{array}{r}2 \\
1 \\
150 \\
1 \\
4\end{array}$ & $\begin{array}{l}\text { 4089. } 23 \\
4088.70 \\
4083.54 \\
4083.20 \\
4079.81\end{array}$ & $\begin{array}{r}1 \\
150 \\
3 \\
1 \\
1 h\end{array}$ & $\begin{array}{r}80 \\
2 \\
\\
1 h\end{array}$ \\
\hline $\begin{array}{l}4687.97 \\
4686.37 \\
4683.58 \\
4678.88 \\
4673.05\end{array}$ & $\begin{array}{l}2 \\
1 \\
1 \\
2 \\
2\end{array}$ & & $\begin{array}{l}\text { 4465. } 61 \\
4463.82 \\
4454.81 \\
4452.24 \\
4434.98\end{array}$ & $\begin{array}{l}2 \\
2 \\
5 \mathrm{Ca} ? \\
2 \\
2\end{array}$ & $\begin{array}{l}1 \\
1 \\
4 \\
1 \\
3\end{array}$ & $\begin{array}{l}\text { 4230. } 22 \\
4229.96 \\
4220.32 \\
4218.61 \\
4215.51\end{array}$ & $\begin{array}{r}1 \\
1 \\
2 \\
30 \\
1\end{array}$ & $\begin{array}{r}1 \\
15 \\
1\end{array}$ & $\begin{array}{l}\text { 4077. } 71 \\
\text { 4069. } 01 \\
\text { 4060. } 84 \\
\text { 4057. } 81 \\
\text { 4057. } 24\end{array}$ & $\begin{array}{l}2 \\
2 \\
1 \\
2 \\
2 h\end{array}$ & $\begin{array}{l}1 \\
1 \\
1 \\
1 h\end{array}$ \\
\hline $\begin{array}{l}4672.82 \\
4672.17 \\
4669.30 \\
4664.32 \\
4660.21\end{array}$ & $\begin{array}{r}2 \\
5 \\
100 \\
2 \\
30\end{array}$ & $\begin{array}{r}40 \\
1 \\
10\end{array}$ & $\begin{array}{l}4432.54 \\
4429.60 \\
4425.47 \\
4420.32 \\
4412.02\end{array}$ & $\begin{array}{c}1 \\
30 \\
1 h \\
1 \\
2\end{array}$ & $\begin{array}{c}15 \\
2 h \\
1\end{array}$ & $\begin{array}{l}\text { 4214. } 22 \\
4212.42 \\
4211.43 \\
4206.58 \\
4201.86\end{array}$ & $\begin{array}{l}1 \\
1 \\
2 \\
9 \\
2\end{array}$ & $\begin{array}{l}2 \\
1 \\
6 \\
1\end{array}$ & $\begin{array}{l}\text { 4056. } 08 \\
4054.42 \\
4053.18 \\
4051.94 \\
4049.10\end{array}$ & $\begin{array}{r}4 h \\
2 h \\
5 \\
5 \\
150\end{array}$ & $\begin{array}{l}3 h \\
1 h \\
3 \\
3 \\
80\end{array}$ \\
\hline $\begin{array}{l}4656.99 \\
4654.64 \\
4653.59 \\
4648.34 \\
4647.57\end{array}$ & $\begin{array}{r}1 \\
2 \\
1 \\
70 \\
2\end{array}$ & $\begin{array}{r}1 \\
20 \\
1\end{array}$ & $\begin{array}{l}\text { 4383. } 10 \\
4373.97 \\
4370.73 \\
4370.10 \\
4369.26\end{array}$ & $\begin{array}{l}2 \\
2 \\
2 \\
2 \\
1\end{array}$ & $\begin{array}{l}1 \\
1 \\
1 \\
1\end{array}$ & $\begin{array}{l}\text { 4199. } 46 \\
4198.31 \\
4189.61 \\
4186.52 \\
4176.28\end{array}$ & $\begin{array}{r}2 \\
1 \\
3 \\
50 \\
100\end{array}$ & $\begin{array}{l}1 \\
4 h \\
2 \\
30 \\
60\end{array}$ & $\begin{array}{l}4041.76 \\
4041.52 \\
4039.23 \\
4037.09 \\
4037.01\end{array}$ & $\begin{array}{r}2 \\
2 \\
10 \\
\\
4 h+g\end{array}$ & $\begin{array}{l}1 \\
1 \\
6 \\
3 h\end{array}$ \\
\hline $\begin{array}{l}4646.61 \\
4645.47 \\
4643.29 \\
4637.51 \\
4635.74\end{array}$ & $\begin{array}{r}1 \\
2 \\
4 \\
90 \\
2\end{array}$ & $\begin{array}{r}1 \\
40 \\
1\end{array}$ & $\begin{array}{l}\text { 4368. } 92 \\
4363.03 \\
4361.95 \\
4359.25 \\
4358.49\end{array}$ & $\begin{array}{l}1 \\
2 \\
1 \\
4 \\
3\end{array}$ & $\begin{array}{l}1 \\
\\
3 \\
2\end{array}$ & $\begin{array}{l}4174.46 \\
4172.53 \\
4170.28 \\
4169.67 \\
4167.43\end{array}$ & $\begin{array}{r}1 \\
80 \\
50 \\
10 \\
6\end{array}$ & $\begin{array}{r}50 \\
30 \\
6 \\
4\end{array}$ & $\begin{array}{l}\text { 4032. } 91 \\
4031.63 \\
4029.08 \\
4026.19 \\
4025.42\end{array}$ & $\begin{array}{c}1 \\
300 \\
1 \\
3 h+g \\
1\end{array}$ & $\begin{array}{c}200 \\
1 \\
2 h\end{array}$ \\
\hline $\begin{array}{l}\text { 4633. } 13 \\
4631.33 \\
4630.57 \\
4624.97 \\
4622.71\end{array}$ & $\begin{array}{r}2 \\
1 \\
20 \\
2 \\
3\end{array}$ & $\begin{array}{r}1 \\
10 \\
1 \\
2\end{array}$ & $\begin{array}{l}\text { 4344. } 58 \\
4342.22 \\
4336.87 \\
4332.46 \\
4331.81\end{array}$ & $\begin{array}{r}2 \\
1 \\
10 \\
3\end{array}$ & $\begin{array}{l}1 \\
\\
5 \\
2 \\
1\end{array}$ & $\begin{array}{l}\text { 4165. } 62 \\
4161.53 \\
4160.02 \\
4153.64 \\
4147.61\end{array}$ & $\begin{array}{r}80 \\
1 \\
1 \\
1 \\
2\end{array}$ & 50 & $\begin{array}{l}\text { 4020. } 77 \\
\text { 4017. } 23 \\
4016.68 \\
4012.01 \\
\text { 4010.08 }\end{array}$ & $\begin{array}{r}10 \\
4 \\
3 \\
6 \\
1\end{array}$ & $\begin{array}{l}7 \\
3 \\
1 \\
4\end{array}$ \\
\hline
\end{tabular}


TABLE 1. Arc and spark spectra of technetium-Continued

\begin{tabular}{|c|c|c|c|c|c|c|c|c|c|c|c|}
\hline \multirow{2}{*}{$\begin{array}{l}\lambda \\
A\end{array}$} & \multicolumn{2}{|c|}{ Intensity } & \multirow{2}{*}{$\begin{array}{l}\lambda \\
A\end{array}$} & \multicolumn{2}{|c|}{ Intensity } & \multirow{2}{*}{$\begin{array}{l}\lambda \\
A\end{array}$} & \multicolumn{2}{|c|}{ Intensity } & \multirow{2}{*}{$\begin{array}{l}\lambda \\
A\end{array}$} & \multicolumn{2}{|c|}{ Intensity } \\
\hline & Arc & Spark & & Arc & Spark & & Arc & Spark & & Are & Spark \\
\hline $\begin{array}{l}4007.13 \\
4004.69 \\
3996.95 \\
3994.51 \\
3994.03\end{array}$ & $\begin{array}{r}4 \\
3 \\
2 \\
40 \\
2\end{array}$ & $\begin{array}{r}3 \\
2 \\
1 \\
30 \\
1\end{array}$ & $\begin{array}{l}3854.05 \\
3852.37 \\
3851.23 \\
3847.59 \\
3847.35\end{array}$ & $\begin{array}{l}1 \\
2 \\
4 \\
7 \\
3\end{array}$ & $\begin{array}{l}1 \\
2 \\
4 \\
1\end{array}$ & $\begin{array}{l}3768.80 \\
3768.35 \\
3763.98 \\
3761.84 \\
3759.19\end{array}$ & $\begin{array}{c}100 \\
1 \\
2 \\
20 \\
1 h\end{array}$ & $\begin{array}{c}60 \\
\\
1 \\
15 \\
2 h\end{array}$ & $\begin{array}{l}\text { 3696. } 74 \\
3696.32 \\
3695.99 \\
3694.34 \\
3692.78\end{array}$ & $\begin{array}{l}4 \\
1 \\
1 \\
6\end{array}$ & $\begin{array}{l}1 h \\
2\end{array}$ \\
\hline $\begin{array}{l}\text { 3992. } 06 \\
\text { 3987. } 77 \\
\text { 3984. } 97 \\
\text { 3982. } 18 \\
\text { 3980. } 36\end{array}$ & $\begin{array}{r}1 \\
3 \\
200 \\
2 \\
4\end{array}$ & $\begin{array}{r}2 \\
100 \\
1 \\
2\end{array}$ & $\begin{array}{l}3845.99 \\
3844.28 \\
3843.98 \\
3841.33 \\
3838.30\end{array}$ & $\begin{array}{l}15 \\
2 h \\
3 \\
10 \\
15 \mathrm{Ca} ?\end{array}$ & $\begin{array}{r}10 \\
1 \\
2 \\
7 \\
10\end{array}$ & $\begin{array}{l}3758.56 \\
3756.28 \\
3755.24 \\
3754.41 \\
3754.09\end{array}$ & $\begin{array}{r}15 \\
1 \\
5 \\
60 \\
3\end{array}$ & $\begin{array}{c}10 \\
1 h \\
3 \\
40 \\
2\end{array}$ & $\begin{array}{l}\text { 3691. } 64 \\
3690.88 \\
3684.78 \\
3683.50 \\
3682.61\end{array}$ & $\begin{array}{c}2 h \\
1 h \\
100 \\
2 \\
3\end{array}$ & $\begin{array}{c}2 h \\
60 \\
1 \\
2\end{array}$ \\
\hline $\begin{array}{l}3979.63 \\
3979.05 \\
3975.02 \\
3971.48 \\
3970.21\end{array}$ & $\begin{array}{l}4 \\
1 \\
5 \\
1\end{array}$ & $\begin{array}{c}2 \\
10 \\
2 h\end{array}$ & $\begin{array}{l}3837.58 \\
3832.82 \\
3832.34 \\
3830.34 \\
3829.28\end{array}$ & $\begin{array}{c}20 \\
5 \\
15 c \\
2 \\
4 d\end{array}$ & $\begin{array}{c}15 \\
3 \\
10 c \\
1 \\
4 d\end{array}$ & $\begin{array}{l}3752.93 \\
3752.16 \\
3749.96 \\
3746.87 \\
3746.18\end{array}$ & $\begin{array}{r}1 \\
30 \\
8 \\
200 \\
15\end{array}$ & $\begin{array}{r}20 \\
5 \\
100 \\
10\end{array}$ & $\begin{array}{l}\text { 3681. } 71 \\
3680.55 \\
3680.34 \\
3679.18 \\
3678.41\end{array}$ & $\begin{array}{r}1 \\
1 \\
10 \\
30 \\
2\end{array}$ & $\begin{array}{c}2 h \\
6 \\
20 \\
1\end{array}$ \\
\hline $\begin{array}{l}\text { 3959. } 93 \\
3959.52 \\
3959.07 \\
3955.74 \\
\text { 3953. } 69\end{array}$ & $\begin{array}{l}3 \\
2 \\
4 \\
3\end{array}$ & $\begin{array}{l}2 h \\
2 \\
1 \\
3 \\
2\end{array}$ & $\begin{array}{l}3828.54 \\
3827.57 \\
3824.46 \\
3819.12 \\
3818.09\end{array}$ & $\begin{array}{l}9 \\
1 \\
6 \\
2 h \\
3\end{array}$ & $\begin{array}{l}6 \\
4 \\
4 h \\
2\end{array}$ & $\begin{array}{l}3745.04 \\
3744.82 \\
3744.04 \\
3742.81 \\
3741.82\end{array}$ & $\begin{array}{l}6 \\
3 \\
4 \\
1\end{array}$ & $\begin{array}{l}4 \\
2 \\
2 \\
3\end{array}$ & $\begin{array}{l}3678.10 \\
3675.88 \\
3675.57 \\
3675.44 \\
3673.27\end{array}$ & $\begin{array}{l}3 \\
1 \\
1\end{array}$ & $\begin{array}{l}2 \\
2 h \\
2 h \\
\\
1 h\end{array}$ \\
\hline $\begin{array}{l}\text { 3951. } 83 \\
3950.38 \\
3947.11 \\
3946.58 \\
3940.51\end{array}$ & $\begin{array}{r}3 \\
2 \\
20 \\
30\end{array}$ & $\begin{array}{c}2 \\
1 \\
15 \\
20 \\
1 h\end{array}$ & $\begin{array}{l}3817.96 \\
3816.90 \\
3815.50 \\
3814.64 \\
3811.28\end{array}$ & $\begin{array}{l}4 \\
6 \\
2 \\
5 \\
3\end{array}$ & $\begin{array}{l}3 \\
4 \\
1 \\
3 \\
2\end{array}$ & $\begin{array}{l}3737.71 \\
3737.44 \\
3736.93 \\
3731.76 \\
3730.85\end{array}$ & $\begin{array}{l}2 h \\
4 \\
4 \\
6 \\
1\end{array}$ & $\begin{array}{l}5 h \\
3 \\
5+\mathrm{Ca} \\
4\end{array}$ & $\begin{array}{l}3669.96 \\
3669.15 \\
3668.96 \\
3667.51 \\
3664.94\end{array}$ & $\begin{array}{l}2 \\
1 \\
1 \\
2 \\
4\end{array}$ & $\begin{array}{l}1 \\
1 \\
\\
1 \\
2\end{array}$ \\
\hline $\begin{array}{l}3040.04 \\
3936.63 \\
3934.82 \\
3929.37 \\
3928.85\end{array}$ & $\begin{array}{l}1 \\
1 \\
1 \\
1 \\
1\end{array}$ & $1 h$ & $\begin{array}{ll}3810.57 \\
3809.78 \\
3808.62 \\
3808.13 \\
3806.13\end{array}$ & $\begin{array}{l}1 \\
1 \\
1 \\
1 \\
1\end{array}$ & 3 & $\begin{array}{l}3729.19 \\
3727.37 \\
3726.36 \\
3726.19 \\
3724.41\end{array}$ & $\begin{array}{c}4 \\
3 \\
100 d \\
2 \\
50\end{array}$ & $\begin{array}{r}3 \\
3 \\
70 \\
1 \\
30\end{array}$ & $\begin{array}{l}3664.58 \\
3662.85 \\
3661.47 \\
3658.62 \\
3654.07\end{array}$ & $\begin{array}{c}1 \\
30 \\
80 \\
2 h\end{array}$ & $\begin{array}{c}2 h \\
\\
20 \\
60 \\
1 h\end{array}$ \\
\hline $\begin{array}{l}3927.57 \\
3923.67 \\
3922.13 \\
3919.38 \\
3917.73\end{array}$ & $\begin{array}{l}2 \\
4 \\
2 \\
6\end{array}$ & $\begin{array}{l}2 \\
3 \\
1 \\
4 \\
1 h\end{array}$ & $\begin{array}{l}3804.00 \\
3801.63 \\
3799.66 \\
3797.79 \\
3797.45\end{array}$ & $\begin{array}{r}2 \\
1 \\
3 \\
30 \\
7\end{array}$ & $\begin{array}{c}1 \\
2 h \\
2 \\
20 \\
4\end{array}$ & $\begin{array}{l}3723.71 \\
3723.13 \\
3718.88 \\
3717.32 \\
3717.04\end{array}$ & $\begin{array}{r}30 \\
1 \\
300 \\
1\end{array}$ & $\begin{array}{r}20 \\
1 \\
150 \\
2\end{array}$ & $\begin{array}{l}3651.50 \\
3650.37 \\
3648.06 \\
3644.15 \\
3643.01\end{array}$ & $\begin{array}{r}10 \\
1 \\
70 \\
2 \\
1\end{array}$ & $\begin{array}{r}8 \\
40 \\
1\end{array}$ \\
\hline $\begin{array}{l}\text { 3916. } 52 \\
3905.76 \\
3905.51 \\
3899.86 \\
3899.11\end{array}$ & $\begin{array}{l}1 \\
1 \\
2 \\
7 \\
1\end{array}$ & $\begin{array}{l}1 \\
4\end{array}$ & $\begin{array}{l}3794.80 \\
3792.10 \\
3791.75 \\
3791.30 \\
3786.56\end{array}$ & $\begin{array}{c}2 \\
1 \\
8 \\
10 \\
1 h\end{array}$ & $\begin{array}{l}6 \\
7 \\
2 h\end{array}$ & $\begin{array}{l}3716.80 \\
3715.96 \\
3714.52 \\
3713.87 \\
3712.84\end{array}$ & $\begin{array}{l}2 \\
9 \\
2 \\
2 g ? \\
5\end{array}$ & $\begin{array}{l}1 \\
6 \\
1 \\
1 \\
3\end{array}$ & $\begin{array}{l}\text { 3641. } 16 \\
3640.23 \\
3639.41 \\
3638.88 \\
3638.24\end{array}$ & $\begin{array}{l}1 h \\
10 \\
30 \\
4 \\
40\end{array}$ & $\begin{array}{l}1 h \\
8 \\
20 \\
3 \\
30\end{array}$ \\
\hline $\begin{array}{ll}3893 . & 23 \\
3892 . & 14 \\
3889 . & 14 \\
3880 . & 74 \\
3879 . & 19\end{array}$ & $\begin{array}{c}2 \\
20 c \\
2 \\
15 \\
10\end{array}$ & $\begin{array}{c}1 \\
40 c \\
1 \\
10 \\
7\end{array}$ & $\begin{array}{l}3786.08 \\
3784.08 \\
3780.70 \\
3779.72 \\
3779.40\end{array}$ & $\begin{array}{r}5 \\
9 \\
70 \\
2 \\
50\end{array}$ & $\begin{array}{r}3 \\
6 \\
50 \\
1 \\
30\end{array}$ & $\begin{array}{l}3712.28 \\
3711.40 \\
3710.13 \\
3709.82 \\
3708.27\end{array}$ & $\begin{array}{r}20 \\
1 \\
2 \\
2 \\
3\end{array}$ & $\begin{array}{r}15 \\
\\
1 \\
1 \\
2\end{array}$ & $\begin{array}{l}\text { 3636. } 10 \\
3635.18 \\
3634.27 \\
3633.28 \\
3630.42\end{array}$ & $\begin{array}{c}400 \\
80 c \\
\\
3 \\
3\end{array}$ & $\begin{array}{c}200 \\
60 c \\
2 h \\
2 \\
2\end{array}$ \\
\hline $\begin{array}{l}3875.64 \\
3869.32 \\
3868.26 \\
3866.16 \\
3865.09\end{array}$ & $\begin{array}{c}3 \\
1 \\
15 \\
1 h \\
1\end{array}$ & $\begin{array}{c}10 \\
3 h\end{array}$ & $\begin{array}{l}3777.30 \\
3776.04 \\
3775.34 \\
3773.43 \\
3772.78\end{array}$ & $\begin{array}{r}15 \\
1 \\
2 \\
3 \\
1\end{array}$ & $\begin{array}{l}1 \\
2\end{array}$ & $\begin{array}{l}3707.65 \\
3706.72 \\
3706.05 \\
3705.70 \\
3705.51\end{array}$ & $\begin{array}{l}3 \\
3 \\
2 \\
1 \\
3\end{array}$ & $\begin{array}{l}2 \\
2 \\
3 h \\
\\
2\end{array}$ & $\begin{array}{l}3628.90 \\
3627.86 \\
3627.38 \\
3625.58 \\
3619.79\end{array}$ & $\begin{array}{r}1 \\
2 \\
30 \\
4 \\
1\end{array}$ & $\begin{array}{c}1 \\
15 \\
3 \\
3 h\end{array}$ \\
\hline $\begin{array}{l}\text { 3864. } 12 \\
\text { 3863. } 84 \\
\text { 3863. } 07 \\
3856.74 \\
3854.34\end{array}$ & $\begin{array}{c}10+\mathrm{Mo} \\
1 \\
4 \\
10 \\
1\end{array}$ & $\begin{array}{l}3 \\
7\end{array}$ & $\begin{array}{l}3772.38 \\
3772.08 \\
3771.44 \\
3771.06 \\
3770.51\end{array}$ & $\begin{array}{r}2 \\
3 \\
1 \\
60 \\
2\end{array}$ & $\begin{array}{r}1 \\
2 \\
40 \\
1\end{array}$ & $\begin{array}{l}\text { 3704. } 83 \\
\text { 3703. } 86 \\
\text { 3703. } 19 \\
\text { 3697. } 76 \\
\text { 3697. } 43\end{array}$ & $\begin{array}{r}6 \\
15 \\
2 \\
1 \\
2\end{array}$ & $\begin{array}{r}5 \\
10 \\
1 \\
1\end{array}$ & $\begin{array}{l}\text { 3619. } 40 \\
3618.96 \\
3616.36 \\
3615.82 \\
3612.85\end{array}$ & $\begin{array}{l}1 \\
4 \\
2\end{array}$ & $\begin{array}{l}2 \\
2 h \\
1 \\
1\end{array}$ \\
\hline
\end{tabular}


TABLE 1. Arc and spark spectra of technetium-Continued

\begin{tabular}{|c|c|c|c|c|c|c|c|c|c|c|c|}
\hline \multirow{2}{*}{$\begin{array}{l}\lambda \\
A\end{array}$} & \multicolumn{2}{|c|}{ Intensity } & \multirow{2}{*}{$\begin{array}{l}\lambda \\
A\end{array}$} & \multicolumn{2}{|c|}{ Intensity } & \multirow{2}{*}{$\begin{array}{l}\lambda \\
A\end{array}$} & \multicolumn{2}{|c|}{ Intensity } & \multirow{2}{*}{$\begin{array}{l}\lambda \\
A\end{array}$} & \multicolumn{2}{|c|}{ Intensity } \\
\hline & Arc & Spark & & Arc & Spark & & Arc & Spark & & Arc & Spark \\
\hline $\begin{array}{l}3612.14 \\
3611.14 \\
3609.90 \\
3609.06 \\
3608.30\end{array}$ & $\begin{array}{c}1 \\
4 \\
2 h \\
1 \\
100\end{array}$ & $\begin{array}{c}1 \\
5 \\
4 h \\
60\end{array}$ & $\begin{array}{l}3525.84 \\
3522.11 \\
3521.29 \\
3519.43 \\
3517.18\end{array}$ & $\begin{array}{r}30 \\
1 \\
1 \\
1 \\
2\end{array}$ & $\begin{array}{l}20 \\
\\
2 \\
1\end{array}$ & $\begin{array}{l}3437.45 \\
3435.70 \\
3434.71 \\
3431.76 \\
3427.87\end{array}$ & $\begin{array}{l}4 \\
1 \\
6 \\
2 \\
1\end{array}$ & $\begin{array}{l}2 \\
\\
3 \\
1\end{array}$ & $\begin{array}{l}\text { 3330. } 79 \\
3327.12 \\
3325.56 \\
3323.90 \\
3322.30\end{array}$ & $\begin{array}{l}3 \\
3 \\
7 \\
\\
2\end{array}$ & $\begin{array}{l}2 \\
2 \\
4 \\
1 h \\
1\end{array}$ \\
\hline $\begin{array}{l}3607.66 \\
3607.35 \\
3604.77 \\
3599.74 \\
3597.43\end{array}$ & $\begin{array}{r}5 \\
50 \\
\\
2 \\
1\end{array}$ & $\begin{array}{c}3 \\
30 \\
1 h \\
1\end{array}$ & $\begin{array}{l}3515.18 \\
3514.29 \\
3510.92 \\
3508.27 \\
3507.19\end{array}$ & $\begin{array}{l}1 \\
5 \\
3 \\
2\end{array}$ & $\begin{array}{l}1 \\
\\
3 \\
2 \\
1\end{array}$ & $\begin{array}{l}3426.66 \\
3425.40 \\
3424.33 \\
3422.97 \\
3421.47\end{array}$ & $\begin{array}{l}1 \\
2 \\
1\end{array}$ & $\begin{array}{l}2 \\
\\
6 \\
2 \\
1\end{array}$ & $\begin{array}{l}3319.27 \\
3318.77 \\
3317.66 \\
3315.81 \\
3313.67\end{array}$ & $\begin{array}{l}2 \\
5\end{array}$ & $\begin{array}{l}1 \\
2 h \\
1 \\
7 \\
3\end{array}$ \\
\hline $\begin{array}{l}3595.70 \\
3594.93 \\
3594.60 \\
3593.49 \\
3591.51\end{array}$ & $\begin{array}{r}50 \\
2 \\
8 \\
9 \\
1\end{array}$ & $\begin{array}{r}30 \\
1 \\
5 \\
5 \\
2\end{array}$ & $\begin{array}{l}3506.13 \\
3505.39 \\
3503.80 \\
3503.58 \\
3503.35\end{array}$ & $\begin{array}{l}2 \\
4 \\
1 \\
1 \\
1\end{array}$ & $\begin{array}{l}1 \\
8\end{array}$ & $\begin{array}{l}3419.12 \\
3418.58 \\
3418.17 \\
3418.07 \\
3417.74\end{array}$ & $\begin{array}{l}6 \\
1 \\
2 \\
2 \\
1\end{array}$ & $\begin{array}{l}3 \\
1 \\
1 \\
1\end{array}$ & $\begin{array}{l}3312.53 \\
3311.04 \\
3310.65 \\
3309.41 \\
3305.91\end{array}$ & $\begin{array}{l}2 \\
2 \\
8 \\
1 h \\
6\end{array}$ & $\begin{array}{l}8 \\
1 \\
5 \\
2 h \\
3\end{array}$ \\
\hline $\begin{array}{l}3591.20 \\
3589.89 \\
3587.96 \\
3585.72 \\
3583.45\end{array}$ & $\begin{array}{c}1 \\
80 \\
2 h \\
1\end{array}$ & $\begin{array}{c}2 h \\
50 \\
1\end{array}$ & $\begin{array}{l}3502.73 \\
3502.49 \\
3501.55 \\
3501.26 \\
3500.72\end{array}$ & $\begin{array}{r}50 \\
2 \\
1 \\
4 \\
20\end{array}$ & $\begin{array}{c}30 \\
1 \\
2 h \\
2 \\
10\end{array}$ & $\begin{array}{l}3411.74 \\
3411.63 \\
3408.31 \\
3407.27 \\
3405.83\end{array}$ & $\begin{array}{l}1 \\
3 \\
2 \\
3 \\
1\end{array}$ & $\begin{array}{l}1 \\
9 \\
1 \\
2\end{array}$ & $\begin{array}{l}\text { 3305. } 61 \\
3302.39 \\
3301.98 \\
3300.78 \\
3298.85\end{array}$ & $\begin{array}{r}1 \\
2 \\
4 \\
3 \\
15\end{array}$ & $\begin{array}{c}2 h \\
1 \\
15 \\
2 \\
60\end{array}$ \\
\hline $\begin{array}{l}3582.66 \\
3582.10 \\
3581.27 \\
3580.08 \\
3579.00\end{array}$ & $\begin{array}{r}50 \\
15 \\
20 \\
20 \\
1\end{array}$ & $\begin{array}{l}30 \\
10 \\
10 \\
15\end{array}$ & $\begin{array}{l}3499.15 \\
3495.84 \\
3494.64 \\
3494.15 \\
3493.41\end{array}$ & $\begin{array}{l}2 \\
1 \\
8 \\
1 \\
8\end{array}$ & $\begin{array}{l}1 \\
5 \\
5\end{array}$ & $\begin{array}{l}3405.34 \\
3403.91 \\
3398.33 \\
3394.20 \\
3392.23\end{array}$ & $\begin{array}{l}3 \\
2 \\
2 \\
8 \\
1\end{array}$ & $\begin{array}{l}2 \\
1 \\
1 \\
5\end{array}$ & $\begin{array}{l}\text { 3298. } 29 \\
\text { 3297. } 36 \\
\text { 3296. } 27 \\
\text { 3293. } 63 \\
3291.96\end{array}$ & $\begin{array}{l}2 \\
1 \\
1\end{array}$ & $\begin{array}{l}2 \\
5 \\
3 \\
1\end{array}$ \\
\hline $\begin{array}{l}3576.50 \\
3576.33 \\
3575.45 \\
3574.61 \\
3574.43\end{array}$ & $\begin{array}{l}1 \\
2 \\
2 \\
1\end{array}$ & $\begin{array}{l}2 h \\
1 \\
1\end{array}$ & $\begin{array}{l}3490.31 \\
3486.25 \\
3484.61 \\
3482.69 \\
3481.40\end{array}$ & $\begin{array}{c}2 \\
50 c \\
1 \\
1 \\
1\end{array}$ & $\begin{array}{c}1 \\
30 \\
\\
2 \\
3 h\end{array}$ & $\begin{array}{l}3391.37 \\
3390.81 \\
3388.18 \\
3386.68 \\
3386.35\end{array}$ & $\begin{array}{l}1 \\
1 \\
1 \\
2 \\
1\end{array}$ & $\begin{array}{l}2 \\
3 \\
3 \\
1\end{array}$ & $\begin{array}{l}\text { 3288. } 30 \\
3287.18 \\
3285.83 \\
3284.77 \\
3282.41\end{array}$ & $\begin{array}{r}5 \\
1 \\
1 \\
20\end{array}$ & $\begin{array}{r}1 \\
10 \\
3 \\
50\end{array}$ \\
\hline $\begin{array}{l}3573.32 \\
3573.07 \\
3572.65 \\
3570.67 \\
3568.87\end{array}$ & $\begin{array}{r}1 \\
1 \\
1 \\
2 \\
20\end{array}$ & $\begin{array}{r}2 \\
\\
3 \\
1 \\
10\end{array}$ & $\begin{array}{l}3475.60 \\
3475.18 \\
3473.72 \\
3472.88 \\
3470.53\end{array}$ & $\begin{array}{r}20 \\
2 \\
1 \\
1 \\
6\end{array}$ & $\begin{array}{r}10 \\
1\end{array}$ & $\begin{array}{l}3385.47 \\
3382.65 \\
3380.39 \\
3378.07 \\
3373.56\end{array}$ & $\begin{array}{l}2 \\
1\end{array}$ & $\begin{array}{l}1 \\
1 h \\
1 \\
2 h\end{array}$ & $\begin{array}{l}3276.30 \\
3272.55 \\
3272.12 \\
3271.19 \\
3270.62\end{array}$ & $\begin{array}{l}2 \\
1 \\
3 \\
2 \\
2\end{array}$ & $\begin{array}{l}1 \\
2 h \\
2 \\
1 \\
1\end{array}$ \\
\hline $\begin{array}{l}3565.43 \\
3565.23 \\
3565.02 \\
3561.23 \\
3560.90\end{array}$ & $\begin{array}{l}3 d \\
1 \\
\\
1 \\
1\end{array}$ & $\begin{array}{l}1 \\
1 h\end{array}$ & $\begin{array}{l}3467.95 \\
3467.21 \\
3466.29 \\
3462.41 \\
3461.68\end{array}$ & $\begin{array}{c}1 h \\
250 c \\
3+\mathrm{Ni}\end{array}$ & $\begin{array}{c}3 h \\
2 h \\
150 \\
2 h \\
1\end{array}$ & $\begin{array}{l}3373.43 \\
3372.80 \\
3371.90 \\
3371.59 \\
3366.76\end{array}$ & $\begin{array}{r}2 \\
1 \\
1 \\
1 \\
10\end{array}$ & 1 & $\begin{array}{l}3270.07 \\
3269.81 \\
3269.68 \\
3269.43 \\
3266.92\end{array}$ & $\begin{array}{l}1 h \\
1 \\
2 \\
1 \\
15\end{array}$ & $\begin{array}{l}2 h \\
4 \\
6 \\
2 h \\
80\end{array}$ \\
\hline $\begin{array}{l}3560.34 \\
3559.77 \\
3556.07 \\
3553.53 \\
3550.66\end{array}$ & $\begin{array}{r}10 \\
5 \\
1 \\
2 \\
100 c\end{array}$ & $\begin{array}{l}6 \\
3 \\
2 h \\
1 \\
70\end{array}$ & $\begin{array}{l}3458.00 \\
3457.59 \\
3457.25 \\
3456.86 \\
3456.48\end{array}$ & $\begin{array}{r}1 \\
2 \\
15 \\
6\end{array}$ & $\begin{array}{l}1 \\
1 \\
8 \\
3 \\
1\end{array}$ & $\begin{array}{l}3363.04 \\
3362.62 \\
3361.20 \\
3359.20 \\
3350.86\end{array}$ & $\begin{array}{l}3 \\
1 \\
1 h \\
2\end{array}$ & $\begin{array}{c}10 \\
1 \\
1 h \\
1\end{array}$ & $\begin{array}{l}3266.31 \\
3264.67 \\
3262.91 \\
3262.12 \\
3261.94\end{array}$ & $\begin{array}{l}2 \\
2 \\
1 \\
1 \\
8\end{array}$ & $\begin{array}{l}6 \\
4 \\
\\
3 \\
5\end{array}$ \\
\hline $\begin{array}{l}\text { 3549. } 74 \\
3541.78 \\
3538.70 \\
3538.13 \\
3535.52\end{array}$ & $\begin{array}{c}150 c \\
50 \\
10 \\
4 \\
10\end{array}$ & $\begin{array}{r}100 \\
30 \\
5 \\
2 \\
8\end{array}$ & $\begin{array}{l}3456.11 \\
3454.08 \\
3453.01 \\
3451.05 \\
3444.00\end{array}$ & $\begin{array}{l}1 \\
1 \\
6\end{array}$ & $\begin{array}{l}4 \\
1 h\end{array}$ & $\begin{array}{l}3350.56 \\
3348.47 \\
3345.60 \\
3339.23 \\
3338.88\end{array}$ & $\begin{array}{l}2 \\
1 \\
8\end{array}$ & $\begin{array}{c}1 \\
2 \\
20 \\
1 \\
2 h\end{array}$ & $\begin{array}{l}3261.39 \\
3259.93 \\
3259.28 \\
3256.34 \\
3256.12\end{array}$ & $\begin{array}{c}3 \\
2 \\
1 h \\
10 \\
4\end{array}$ & $\begin{array}{r}2 \\
1 \\
50 \\
3\end{array}$ \\
\hline $\begin{array}{l}3534.87 \\
3531.34 \\
3529.85 \\
3527.96 \\
3526.19\end{array}$ & $\begin{array}{l}3 \\
1 \\
2 \\
1 \\
7\end{array}$ & $\begin{array}{l}2 \\
1 \\
4\end{array}$ & $\begin{array}{l}3443.49 \\
3442.25 \\
3441.09 \\
3438.74 \\
3438.24\end{array}$ & $\begin{array}{l}3 \\
\\
1 h \\
2\end{array}$ & $\begin{array}{l}2 \\
2 h \\
3 h \\
1 \\
2 h\end{array}$ & $\begin{array}{l}3338.27 \\
3337.55 \\
3334.53 \\
3332.48 \\
3331.75\end{array}$ & $\begin{array}{l}2 \\
2\end{array}$ & $\begin{array}{l}1 h \\
1 \\
1 \\
1 \\
1 h\end{array}$ & $\begin{array}{l}3254.96 \\
3254.70 \\
3252.06 \\
3250.39 \\
3249.31\end{array}$ & $\begin{array}{r}1 \\
1 \\
10\end{array}$ & $\begin{array}{l}4 \\
8 \\
1 \\
1\end{array}$ \\
\hline
\end{tabular}


TABLE 1. Arc and spark spectra of technetium-Continued

\begin{tabular}{|c|c|c|c|c|c|c|c|c|c|c|c|}
\hline \multirow{2}{*}{$\begin{array}{l}\lambda \\
A\end{array}$} & \multicolumn{2}{|c|}{ Intensity } & \multirow{2}{*}{$\begin{array}{l}\lambda \\
A\end{array}$} & \multicolumn{2}{|c|}{ Intensity } & \multirow{2}{*}{$\begin{array}{l}\lambda \\
A\end{array}$} & \multicolumn{2}{|c|}{ Intensity } & \multirow{2}{*}{$\begin{array}{l}\lambda \\
A\end{array}$} & \multicolumn{2}{|c|}{ Intensity } \\
\hline & Are & Spark & & Are & Spark & & Arc & Spark & & Arc & Spark \\
\hline $\begin{array}{l}3244.19 \\
3242.29 \\
3241.85 \\
3240.68 \\
3239.79\end{array}$ & $\begin{array}{r}10 \\
1 \\
3 \\
1\end{array}$ & $\begin{array}{l}8 \\
1 \\
2 \\
1 \\
1\end{array}$ & $\begin{array}{l}3156.81 \\
3153.35 \\
3153.17 \\
3150.26 \\
3146.24\end{array}$ & $\begin{array}{l}1 \\
1 \\
1 \\
2 \\
1\end{array}$ & $\begin{array}{l}2 \\
\\
2 \\
1 \\
1\end{array}$ & $\begin{array}{l}3062.39 \\
3062.13 \\
3061.78 \\
3060.91 \\
3060.69\end{array}$ & $\begin{array}{l}3 \\
2\end{array}$ & $\begin{array}{l}2 \\
1 \\
2 h \\
2 h \\
1\end{array}$ & $\begin{array}{l}\text { 2988. } 61 \\
2988.20 \\
2987.23 \\
2985.38 \\
2985.05\end{array}$ & $\begin{array}{l}1 \\
3 \\
1\end{array}$ & $\begin{array}{l}2 \mathrm{Ca} ? \\
1 h \\
3\end{array}$ \\
\hline $\begin{array}{l}3239.51 \\
3238.50 \\
3237.02 \\
3235.00 \\
3230.95\end{array}$ & $\begin{array}{c}1 \\
200 c \\
5\end{array}$ & $\begin{array}{c}1 \\
1 \\
400 c \\
1 \\
15\end{array}$ & $\begin{array}{l}3146.00 \\
3145.62 \\
3144.58 \\
3144.17 \\
3143.63\end{array}$ & $\begin{array}{l}1 \\
1 \\
1\end{array}$ & $\begin{array}{l}1 \\
3 h \\
2 \\
2 \\
2 h\end{array}$ & $\begin{array}{l}3058.52 \\
3057.52 \\
3057.34 \\
3057.04 \\
3055.47\end{array}$ & $\begin{array}{l}1 \\
2 \\
2\end{array}$ & $\begin{array}{l}4 \\
2 \\
1 \\
1 \\
1 h\end{array}$ & $\begin{array}{l}2984.40 \\
2984.15 \\
2983.73 \\
2982.55 \\
2980.84\end{array}$ & $\begin{array}{r}5 \\
2 \\
1 \\
10\end{array}$ & $\begin{array}{c}15 \\
1 \\
2 h \\
40 \\
1 h\end{array}$ \\
\hline $\begin{array}{l}3230.02 \\
3226.05 \\
3223.59 \\
3220.73 \\
3219.73\end{array}$ & $\begin{array}{l}3 \\
2 \\
1 \\
3 \\
2\end{array}$ & $\begin{array}{l}2 \\
1 \\
3 \\
2 \\
5\end{array}$ & $\begin{array}{l}3141.00 \\
3140.67 \\
3139.99 \\
3138.33 \\
3136.61\end{array}$ & $\begin{array}{l}1 h \\
1 h \\
1 \\
1 \\
2\end{array}$ & $\begin{array}{l}3 h \\
2 h \\
1 h \\
1 \\
10\end{array}$ & $\begin{array}{l}3053.10 \\
3052.50 \\
3051.57 \\
3049.05 \\
3047.55\end{array}$ & $\begin{array}{l}2 \\
3 \\
\\
2\end{array}$ & $\begin{array}{l}1 h \\
3 \\
2 \\
1 h \\
3\end{array}$ & $\begin{array}{l}2979.76 \\
2976.57 \\
2976.19 \\
2973.67 \\
2970.76\end{array}$ & $\begin{array}{r}2 \\
15 \\
2 \\
4 \\
1 h\end{array}$ & $\begin{array}{c}3 \\
40 \\
5 \\
3 \\
6 H l\end{array}$ \\
\hline $\begin{array}{l}3218.58 \\
3218.25 \\
3217.48 \\
3217.12 \\
3216.53\end{array}$ & $\begin{array}{l}1 \\
1 \\
2 \\
1 \\
1\end{array}$ & $\begin{array}{l}3 \\
1 \\
2\end{array}$ & $\begin{array}{l}3133.35 \\
3133.15 \\
3131.26 \\
3127.87 \\
3122.66\end{array}$ & $\begin{array}{r}1 \\
15 \\
1 \\
6\end{array}$ & $\begin{array}{l}1 \\
4 \\
8 \\
\\
3\end{array}$ & $\begin{array}{l}\text { 3046. } 53 \\
3045.30 \\
3044.71 \\
\text { 3043. } 78 \\
\text { 3043. } 08\end{array}$ & $\begin{array}{l}1 \\
1 \\
1 \\
1\end{array}$ & $\begin{array}{l}4 \\
2 h \\
3\end{array}$ & $\begin{array}{l}2968.79 \\
2967.13 \\
2966.57 \\
2966.19 \\
2965.49\end{array}$ & $\begin{array}{l}1 \\
3 h l \\
1 \\
3\end{array}$ & $\begin{array}{c}10 H l \\
3 \\
8 \\
1\end{array}$ \\
\hline $\begin{array}{l}3215.80 \\
3214.90 \\
3213.29 \\
3212.01 \\
3208.87\end{array}$ & $\begin{array}{c}1 \\
1 \\
1 \\
80 c \\
1 h\end{array}$ & $\begin{array}{r}1 \\
1 \\
1 \\
300 c \\
1 h\end{array}$ & $\begin{array}{l}3121.97 \\
3119.90 \\
3119.68 \\
3119.18 \\
3116.90\end{array}$ & $\begin{array}{l}3 \\
2 \\
2 \\
3 \\
1\end{array}$ & $\begin{array}{l}6 \\
5 \\
1 \\
2\end{array}$ & $\begin{array}{l}\text { 3042. } 66 \\
3040.90 \\
3040.63 \\
\text { 3038. } 24 \\
\text { 3037. } 91\end{array}$ & $\begin{array}{l}2+\mathrm{Fe} \\
1 \\
1 \\
3 \\
15\end{array}$ & $\begin{array}{r}1 \\
1 \\
1 \\
2 \\
50\end{array}$ & $\begin{array}{l}2964.50 \\
2962.58 \\
2962.09 \\
2961.63 \\
2958.88\end{array}$ & $\begin{array}{l}20 \\
\\
3 \\
2 \\
1 h\end{array}$ & $\begin{array}{l}60 \\
1 \\
9 \\
6 \\
2 h\end{array}$ \\
\hline $\begin{array}{l}3202.84 \\
3200.98 \\
3198.49 \\
3197.72 \\
3197.53\end{array}$ & $\begin{array}{l}8 \\
1 \\
2 \\
2 \\
3\end{array}$ & $\begin{array}{l}5 \\
\\
1 \\
1 \\
2\end{array}$ & $\begin{array}{l}3116.01 \\
3115.31 \\
3110.59 \\
3109.14 \\
3106.95\end{array}$ & $\begin{array}{l}2 \\
\\
1 \\
2 \\
2\end{array}$ & $\begin{array}{l}1 \\
1 \\
4 \\
5 \\
1\end{array}$ & $\begin{array}{l}\text { 3036. } 90 \\
\text { 3034. } 61 \\
\text { 3033. } 64 \\
\text { 3033. } 18 \\
3032.07\end{array}$ & $\begin{array}{l}2 \\
2 \\
\\
2 \\
1\end{array}$ & $\begin{array}{l}1 \\
1 \\
1 \\
1 \\
2\end{array}$ & $\begin{array}{l}\text { 2958. } 48 \\
2958.38 \\
2957.83 \\
2956.25 \\
2955.94\end{array}$ & $\begin{array}{l}1 \\
2 \\
4\end{array}$ & $\begin{array}{l}1 \\
2 \\
1 \\
1 \\
2\end{array}$ \\
\hline $\begin{array}{l}3195.68 \\
3195.21 \\
3192.35 \\
3192.07 \\
3191.63\end{array}$ & $\begin{array}{r}50 \\
\\
2 \\
1\end{array}$ & $\begin{array}{r}2 \\
200 \\
1 \\
1\end{array}$ & $\begin{array}{l}3105.39 \\
3105.13 \\
3104.29 \\
3100.89 \\
3099.54\end{array}$ & $\begin{array}{l}1 \\
2 \\
1 \\
2 \\
4\end{array}$ & $\begin{array}{l}2 \\
8 \\
\\
5 \\
2\end{array}$ & $\begin{array}{l}3029.22 \\
3026.89 \\
3026.44 \\
\text { 3025. } 28 \\
\text { 3023. } 71\end{array}$ & $\begin{array}{r}10 \\
\\
3 \\
4\end{array}$ & $\begin{array}{r}1 \\
20 \\
1 \\
2 \\
3\end{array}$ & $\begin{array}{l}2953.18 \\
2952.55 \\
2952.07 \\
2951.05 \\
2950.81\end{array}$ & $\begin{array}{l}1 \\
2 \\
1 \\
1 \\
3\end{array}$ & $\begin{array}{l}2 \\
6 \\
3 \\
2 \\
6\end{array}$ \\
\hline $\begin{array}{l}3190.36 \\
3189.71 \\
3188.00 \\
3183.12 \\
3182.80\end{array}$ & $\begin{array}{r}30 \\
1\end{array}$ & $\begin{array}{c}20 \\
1 h \\
1 h \\
15 \\
1\end{array}$ & $\begin{array}{l}\text { 3099. } 12 \\
\text { 3098. } 58 \\
\text { 3097. } 28 \\
\text { 3095. } 77 \\
\text { 3093. } 64\end{array}$ & $\begin{array}{l}6 \\
2 \\
1 \\
2\end{array}$ & $\begin{array}{l}3 \\
5 \\
4 \\
2 d \\
2\end{array}$ & $\begin{array}{l}\text { 3022. } 22 \\
3022.06 \\
3019.19 \\
3017.25 \\
3016.31\end{array}$ & $\begin{array}{l}1 \\
1 \\
2 \\
6 \\
1\end{array}$ & $\begin{array}{l}3 \\
4 \\
4\end{array}$ & $\begin{array}{l}2950.40 \\
2950.26 \\
2949.58 \\
2949.48 \\
2949.25\end{array}$ & $\begin{array}{l}4 \\
1 \\
1 \\
1 \\
2\end{array}$ & $\begin{array}{c}10 \\
3 \\
\\
2 h \\
1\end{array}$ \\
\hline $\begin{array}{l}3182.38 \\
3181.31 \\
3180.31 \\
3178.34 \\
3177.91\end{array}$ & $\begin{array}{r}30 \\
1 \\
5 \\
1\end{array}$ & $\begin{array}{c}15 \\
2 h \\
3 \\
1 \\
2\end{array}$ & $\begin{array}{l}\text { 3090. } 26 \\
3089.36 \\
3084.51 \\
3083.31 \\
3081.10\end{array}$ & $\begin{array}{l}2 \\
3 \\
\\
2 d \\
1\end{array}$ & $\begin{array}{l}1 \\
2 \\
1 h \\
4 d \\
1\end{array}$ & $\begin{array}{l}3015.16 \\
3009.76 \\
3008.78 \\
3007.00 \\
3005.51\end{array}$ & $\begin{array}{l}4 \\
1\end{array}$ & $\begin{array}{r}5 \\
1 \\
1 \\
15 \\
2\end{array}$ & $\begin{array}{l}2948.64 \\
2948.48 \\
2948.10 \\
2947.12 \\
2946.58\end{array}$ & $\begin{array}{r}20 \\
10 \\
1 \\
2 \\
1\end{array}$ & $\begin{array}{r}60 \\
20 \\
2 \\
1 \\
2\end{array}$ \\
\hline $\begin{array}{l}3174.75 \\
3173.30 \\
3171.94 \\
3171.47 \\
3170.38\end{array}$ & $\begin{array}{c}1 \\
40 \\
2 d \\
1 \\
2\end{array}$ & $\begin{array}{l}3 \\
20 \\
2 d \\
2 H l \\
6\end{array}$ & $\begin{array}{l}3079.92 \\
3077.70 \\
3076.27 \\
3074.31 \\
3072.92\end{array}$ & $\begin{array}{l}1 \\
3 \\
3 \\
2 \\
1\end{array}$ & $\begin{array}{r}3 \\
10 \\
2 \\
1 \\
2\end{array}$ & $\begin{array}{l}\text { 3004. } 62 \\
3004.40 \\
3003.71 \\
3003.03 \\
3001.05\end{array}$ & $\begin{array}{l}1 \\
2 \\
5\end{array}$ & $\begin{array}{r}1 \\
3 \\
1 \\
2 \\
15\end{array}$ & $\begin{array}{l}2946.21 \\
2945.53 \\
2942.92 \\
2941.84 \\
2940.92\end{array}$ & $\begin{array}{r}1 \\
10\end{array}$ & $\begin{array}{r}1 \\
3 \\
30 \\
2 \\
2\end{array}$ \\
\hline $\begin{array}{l}3169.27 \\
3165.15 \\
3161.70 \\
3158.07 \\
3157.66\end{array}$ & $\begin{array}{l}1 \\
5 \\
1\end{array}$ & $\begin{array}{l}1 \\
4 \\
3 \\
2 \\
1\end{array}$ & $\begin{array}{l}3070.28 \\
3069.97 \\
3068.34 \\
3066.61 \\
3066.40\end{array}$ & $\begin{array}{l}2 \\
5 \\
2 \\
1\end{array}$ & $\begin{array}{r}1 \\
20 \\
1 \\
1\end{array}$ & $\begin{array}{l}\text { 2996. } 88 \\
\text { 2994. } 12 \\
\text { 2993. } 94 \\
\text { 2993. } 77 \\
\text { 2992. } 87\end{array}$ & $\begin{array}{l}1 \\
1 \\
1 \\
1 h\end{array}$ & $\begin{array}{l}1 \\
2 \\
1 \\
3\end{array}$ & $\begin{array}{l}2940.49 \\
2940.01 \\
2939.25 \\
2938.98 \\
2936.93\end{array}$ & $\begin{array}{r}2 \\
10 \\
4\end{array}$ & $\begin{array}{r}1 \\
1 \\
1 \\
20 \\
15\end{array}$ \\
\hline
\end{tabular}


TABLE 1. Arc and spark spectra of technetium-Continued

\begin{tabular}{|c|c|c|c|c|c|c|c|c|c|c|c|}
\hline \multirow{2}{*}{$\begin{array}{l}\lambda \\
A\end{array}$} & \multicolumn{2}{|c|}{ Intensity } & \multirow{2}{*}{$\begin{array}{l}\lambda \\
A\end{array}$} & \multicolumn{2}{|c|}{ Intensity } & \multirow{2}{*}{$\begin{array}{l}\lambda \\
A\end{array}$} & \multicolumn{2}{|c|}{ Intensity } & \multirow{2}{*}{$\begin{array}{l}\lambda \\
A\end{array}$} & \multicolumn{2}{|c|}{ Intensity } \\
\hline & Arc & Spark & & Arc & Spark & & Are & Spark & & Arc & Spark \\
\hline $\begin{array}{l}\text { 2934. } 65 \\
\text { 2933. } 94 \\
\text { 2932. } 94 \\
\text { 2932. } 12 \\
\text { 2931. } 56\end{array}$ & $\begin{array}{r}15 \\
1 \\
1 \\
1\end{array}$ & $\begin{array}{r}1 \\
30 \\
2 \\
3 \\
2\end{array}$ & $\begin{array}{l}\text { 2890. } 47 \\
2889.23 \\
\text { 2888. } 48 \\
\text { 2887. } 76 \\
2887.43\end{array}$ & $\begin{array}{r}2 \\
30 \\
10 \\
20\end{array}$ & $\begin{array}{r}6 \\
80 \\
10 \\
10 \\
1\end{array}$ & $\begin{array}{l}2845.06 \\
2843.78 \\
2843.35 \\
2842.30 \\
2840.38\end{array}$ & $\begin{array}{r}5 \\
1 \\
1 \\
30\end{array}$ & $\begin{array}{r}3 \\
1 \\
3 \\
2 \\
100\end{array}$ & $\begin{array}{l}\text { 2800. } 79 \\
\text { 2800. } 46 \\
\text { 2797. } 75 \\
\text { 2796. } 72 \\
\text { 2795. } 78\end{array}$ & $\begin{array}{r}2 \\
1 \\
3 \\
15 \\
100\end{array}$ & $\begin{array}{r}6 \\
3 \\
6 \\
30 \\
200\end{array}$ \\
\hline $\begin{array}{l}2931.14 \\
2930.50 \\
2930.05 \\
2929.53 \\
2928.45\end{array}$ & $\begin{array}{l}3 \\
3 \\
2 \\
2 \\
4\end{array}$ & $\begin{array}{r}8 \\
2 \\
5 \\
8 \\
10\end{array}$ & $\begin{array}{l}\text { 2886. } 10 \\
2885.55 \\
2884.76 \\
2883.51 \\
2882.39\end{array}$ & $\begin{array}{l}2 \\
2 h \\
1 \\
5\end{array}$ & $\begin{array}{l}5 \\
6 h \\
2 \\
1 \\
10\end{array}$ & $\begin{array}{l}2839.87 \\
2838.88 \\
2838.56 \\
2836.91 \\
2836.13\end{array}$ & $\begin{array}{r}2 \\
1 \\
8 \\
10\end{array}$ & $\begin{array}{c}10 \\
3 \\
15 \\
1 h \\
30\end{array}$ & $\begin{array}{l}\text { 2795. } 08 \\
2794.55 \\
2794.24 \\
2793.67 \\
2792.98\end{array}$ & $\begin{array}{r}2 \\
4 \\
15\end{array}$ & $\begin{array}{r}4 \\
2 \\
30 \\
1 \\
2\end{array}$ \\
\hline $\begin{array}{l}\text { 2928. } 20 \\
2927.32 \\
2925.10 \\
2924.87 \\
2924.27\end{array}$ & $\begin{array}{r}10 \\
1 \\
1 \\
1 \\
3\end{array}$ & $\begin{array}{r}5 \\
3 \\
3 \\
1 \\
10\end{array}$ & $\begin{array}{l}2881.32 \\
2881.15 \\
2880.43 \\
2879.15 \\
2878.70\end{array}$ & $\begin{array}{r}2 \\
20 \\
4 \\
3\end{array}$ & $\begin{array}{l}2 h \\
2 \\
60 \\
10 \\
8\end{array}$ & $\begin{array}{l}2835.63 \\
2834.75 \\
2834.32 \\
2833.60 \\
2831.19\end{array}$ & $\begin{array}{r}2 \\
1 \\
\\
15 \\
30\end{array}$ & $\begin{array}{r}1 \\
2 \\
2 \\
40 \\
100\end{array}$ & $\begin{array}{l}2792.09 \\
2790.46 \\
2790.28 \\
2789.27 \\
2788.90\end{array}$ & $\begin{array}{l}1 h \\
2 \\
1 \\
20 \\
2\end{array}$ & $\begin{array}{c}2 h \\
2 \\
1 \\
40 \\
3\end{array}$ \\
\hline $\begin{array}{l}\text { 2923. } 35 \\
\text { 2922. } 75 \\
2921.94 \\
2921.52 \\
2921.07\end{array}$ & $\begin{array}{r}20 \\
10 \\
8 \\
4 \\
15\end{array}$ & $\begin{array}{r}40 \\
20 \\
4 \\
15 \\
60\end{array}$ & $\begin{array}{l}2877.18 \\
2874.12 \\
2873.93 \\
2873.68 \\
2872.24\end{array}$ & $\begin{array}{l}2 \\
2 \\
5 c w\end{array}$ & $\begin{array}{c}1 \\
4 \\
1 \\
1 \\
15 c w\end{array}$ & $\begin{array}{l}2830.87 \\
2829.62 \\
2829.09 \\
2828.07 \\
2826.18\end{array}$ & $\begin{array}{l}1 \\
1 h \\
1 \\
15 \\
10\end{array}$ & $\begin{array}{l}3 \\
2 h \\
2 \\
8 \\
30\end{array}$ & $\begin{array}{l}2788.81 \\
2787.95 \\
2787.65 \\
2787.26 \\
2785.60\end{array}$ & $\begin{array}{r}1 \\
2 \\
2 \\
15\end{array}$ & $\begin{array}{l}2 \\
6 \\
1 \\
1 \\
7\end{array}$ \\
\hline $\begin{array}{l}\text { 2919. } 66 \\
\text { 2915. } 86 \\
\text { 2914. } 24 \\
\text { 2913. } 81 \\
\text { 2913. } 17\end{array}$ & $\begin{array}{r}1 \\
15\end{array}$ & $\begin{array}{l}1 \\
1 \\
1 h \\
1 \\
10\end{array}$ & $\begin{array}{l}2871.98 \\
2871.45 \\
2870.43 \\
2870.31 \\
2869.51\end{array}$ & $\begin{array}{l}1 \\
1 \\
2 \\
2\end{array}$ & $\begin{array}{l}2 \\
2 h \mathrm{Cr} ? \\
6 \mathrm{Cr} ? \\
2 \\
1\end{array}$ & $\begin{array}{l}2825.98 \\
2825.35 \\
2825.06 \\
2822.92 \\
2821.62\end{array}$ & $\begin{array}{l}2 \\
15 \\
10 \\
1 h \\
10\end{array}$ & $\begin{array}{c}1 \\
40 \\
30 \\
2 h \\
20\end{array}$ & $\begin{array}{l}2785.30 \\
2785.15 \\
2784.74 \\
2784.39 \\
2782.08\end{array}$ & $\begin{array}{r}2 \\
1 \\
1 \\
30\end{array}$ & $\begin{array}{r}5 \\
2 \\
1 \\
3 \\
15\end{array}$ \\
\hline $\begin{array}{l}\text { 2912. } 01 \\
\text { 2911. } 71 \\
2910.70 \\
2910.23 \\
\text { 2909. } 09\end{array}$ & $\begin{array}{r}2 \\
1 \\
1 \\
20 \\
3\end{array}$ & $\begin{array}{r}4 \\
2 \\
2 \\
40 \\
10\end{array}$ & $\begin{array}{l}2869.30 \\
2868.81 \\
2868.11 \\
2867.22 \\
2866.09\end{array}$ & $\begin{array}{c}6 \\
1 \\
5 \\
1 \\
20 c\end{array}$ & $\begin{array}{r}10 \\
1 \\
3 \\
\\
60 c\end{array}$ & $\begin{array}{l}2821.37 \\
2820.19 \\
2819.48 \\
2819.06 \\
2818.65\end{array}$ & $\begin{array}{r}30 \\
2 \\
2 \\
1 \\
2\end{array}$ & $\begin{array}{r}100 \\
6 \\
1 \\
2 \\
6\end{array}$ & $\begin{array}{l}2781.24 \\
2779.81 \\
2778.93 \\
2778.13 \\
2777.65\end{array}$ & $\begin{array}{l}2 \\
10 \mathrm{Mg} ? \\
5 \\
2\end{array}$ & $\begin{array}{r}1 \\
15 \\
6 \\
4 \\
1\end{array}$ \\
\hline $\begin{array}{l}2907.99 \\
2907.63 \\
2906.90 \\
2906.63 \\
2906.26\end{array}$ & $\begin{array}{l}2 \\
2 h \\
1 h \\
6 \\
1\end{array}$ & $\begin{array}{c}4 h \\
2 h \\
20 \\
2\end{array}$ & $\begin{array}{l}2864.91 \\
2864.51 \\
2863.54 \\
2863.35 \\
2862.77\end{array}$ & $\begin{array}{r}10 \\
2\end{array}$ & $\begin{array}{l}2 \\
5 \\
1 \\
1\end{array}$ & $\begin{array}{l}2817.62 \\
2817.29 \\
2817.01 \\
2816.51 \\
2815.97\end{array}$ & $\begin{array}{l}3 \\
1 \\
5 \\
2 \\
2\end{array}$ & $\begin{array}{r}7 \\
2 \\
10 \\
6 \\
4\end{array}$ & $\begin{array}{l}2777.33 \\
2776.57 \\
2776.06 \\
2775.61 \\
2775.08\end{array}$ & $\begin{array}{r}40 \\
1 \\
2 \\
10 \\
1\end{array}$ & $\begin{array}{r}100 \\
2 \\
4 \\
30 \\
2\end{array}$ \\
\hline $\begin{array}{l}\text { 2904. } 96 \\
\text { 2903. } 84 \\
\text { 2902. } 93 \\
\text { 2902. } 54 \\
\text { 2902. } 15\end{array}$ & $\begin{array}{r}1 \\
3 \\
1 \\
1 \\
15\end{array}$ & $\begin{array}{r}2 \\
2 \\
2 \\
40\end{array}$ & $\begin{array}{l}\text { 2861. } 70 \\
2861.35 \\
2860.98 \\
2860.78 \\
2859.13\end{array}$ & $\begin{array}{r}2 \\
\\
2 \\
30 \\
40\end{array}$ & $\begin{array}{r}8 \\
1 \\
1 \\
60 \\
40\end{array}$ & $\begin{array}{l}2814.99 \\
2814.88 \\
2814.64 \\
2814.20 \\
2813.80\end{array}$ & $\begin{array}{r}2 \\
20 \\
2 \\
15 \\
10\end{array}$ & $\begin{array}{r}4 \\
10 \\
4 \\
30 \\
20\end{array}$ & $\begin{array}{l}\text { 2774. } 29 \\
2773.79 \\
2773.32 \\
2772.06 \\
2771.80\end{array}$ & $\begin{array}{r}1 \\
15 \\
2 \\
1 \\
1\end{array}$ & $\begin{array}{r}2 \\
50 \\
6 \\
2 \\
3\end{array}$ \\
\hline $\begin{array}{l}\text { 2900. } 69 \\
2899.37 \\
2896.58 \\
2896.36 \\
2895.48\end{array}$ & $\begin{array}{r}5 \\
1 \\
15 \\
2\end{array}$ & $\begin{array}{r}15 \\
1 \\
2 \\
10 \\
5\end{array}$ & $\begin{array}{l}2857.15 \\
2855.66 \\
2853.64 \\
2853.28 \\
2851.23\end{array}$ & $\begin{array}{r}15 \\
2\end{array}$ & $\begin{array}{c}10 \\
2 \\
1 h \\
1 \\
1\end{array}$ & $\begin{array}{l}2813.50 \\
2813.00 \\
2811.62 \\
2811.15 \\
2810.24\end{array}$ & $\begin{array}{c}2 d \\
1 \\
80 \\
\\
4\end{array}$ & $\begin{array}{c}3 d \\
3 \\
200 \\
1 h \\
8\end{array}$ & $\begin{array}{l}2771.22 \\
2769.07 \\
2768.40 \\
2768.10 \\
2767.34\end{array}$ & $\begin{array}{c}10 c \\
1 \\
1 \\
1 h\end{array}$ & $\begin{array}{c}30 c \\
2 \\
2 \\
1 \\
3 h\end{array}$ \\
\hline $\begin{array}{l}\text { 2894. } 83 \\
\text { 2894. } 34 \\
\text { 2893. } 90 \\
\text { 2893. } 47 \\
\text { 2893. } 18\end{array}$ & $\begin{array}{l}2 \\
6 \\
1 \\
5 \\
5\end{array}$ & $\begin{array}{l}5 \\
3 \\
3 \\
2 \\
2\end{array}$ & $\begin{array}{l}2850.97 \\
2850.18 \\
2849.21 \\
2847.88 \\
2847.68\end{array}$ & $\begin{array}{l}8 \\
4\end{array}$ & $\begin{array}{l}8 \\
1 \\
3 \\
1 \\
1\end{array}$ & $\begin{array}{l}2810.06 \\
2809.66 \\
2808.38 \\
2807.93 \\
2807.43\end{array}$ & $\begin{array}{c}1 \\
20 c \\
15 \\
3 d\end{array}$ & $\begin{array}{c}60 c \\
8 \\
6 d \\
1 h\end{array}$ & $\begin{array}{l}2766.90 \\
2765.98 \\
2765.73 \\
2765.52 \\
2764.51\end{array}$ & $\begin{array}{r}10 \\
3 \\
3\end{array}$ & $\begin{array}{l}5 \\
1 \\
7 \\
1 \\
1\end{array}$ \\
\hline $\begin{array}{l}2892.78 \\
2892.35 \\
2891.34 \\
2891.20 \\
2890.81\end{array}$ & $\begin{array}{r}10 \\
5 \\
2 \\
1 \\
20 c\end{array}$ & $\begin{array}{r}30 \\
20 \\
5 \\
3 \\
60 c\end{array}$ & $\begin{array}{l}2846.74 \\
2846.62 \\
2846.40 \\
2845.78 \\
2845.46\end{array}$ & $\begin{array}{r}5 \\
1 \\
15 \\
1\end{array}$ & $\begin{array}{r}15 \\
3 \\
50 \\
1 \\
2\end{array}$ & $\begin{array}{l}2807.09 \\
2806.65 \\
2805.19 \\
2803.35 \\
2803.03\end{array}$ & $\begin{array}{l}2 \\
1 \\
5\end{array}$ & $\begin{array}{c}1 h \\
5 \\
3 \\
1 \\
10 ?\end{array}$ & $\begin{array}{l}2763.25 \\
2762.36 \\
2762.16 \\
2761.29 \\
2760.40\end{array}$ & $\begin{array}{r}2 \\
10 \\
7 \\
1 \\
1\end{array}$ & $\begin{array}{l}8 \\
5 \\
3 \\
3\end{array}$ \\
\hline
\end{tabular}


TABLE 1. Arc and spark spectra of technetıum-Continued

\begin{tabular}{|c|c|c|c|c|c|c|c|c|c|c|c|}
\hline \multirow{2}{*}{$\begin{array}{l}\lambda \\
A\end{array}$} & \multicolumn{2}{|c|}{ Intensity } & \multirow{2}{*}{$\begin{array}{l}\lambda \\
A\end{array}$} & \multicolumn{2}{|c|}{ Intensity } & \multirow{2}{*}{$\begin{array}{l}\lambda \\
A\end{array}$} & \multicolumn{2}{|c|}{ Intensity } & \multirow{2}{*}{$\begin{array}{l}\lambda \\
A\end{array}$} & \multicolumn{2}{|c|}{ Intensity } \\
\hline & Arc & Spark & & Arc & Spark & & Arc & Spark & & Arc & Spark \\
\hline $\begin{array}{l}2760.13 \\
2758.72 \\
2757.58 \\
2757.25 \\
2757.01\end{array}$ & $\begin{array}{l}1 h \\
1 \\
2 \\
1\end{array}$ & $\begin{array}{l}3 h \\
2 \\
3 \\
4 \\
2\end{array}$ & $\begin{array}{l}2714.67 \\
2714.58 \\
2712.62 \\
2711.94 \\
2711.50\end{array}$ & $\begin{array}{c}2 \\
3 \\
10 \\
1 h \\
2\end{array}$ & $\begin{array}{c}4 \\
6 \\
30 \\
2 h \\
6\end{array}$ & $\begin{array}{l}2671.02 \\
2670.84 \\
2670.12 \\
2669.85 \\
2669.67\end{array}$ & $\begin{array}{l}2 \\
1 \\
4 \\
1 \\
1\end{array}$ & $\begin{array}{l}6 \\
2 \\
7 \\
2 h \\
2 h\end{array}$ & $\begin{array}{l}2635.77 \\
2634.92 \\
2630.30 \\
2629.52 \\
2627.97\end{array}$ & $\begin{array}{r}1 \\
150\end{array}$ & $\begin{array}{c}3 \\
300 \\
1 h \\
1 h \\
15 c\end{array}$ \\
\hline $\begin{array}{l}2756.50 \\
2755.77 \\
2753.06 \\
2752.30 \\
2751.49\end{array}$ & $\begin{array}{r}10 \\
1 \\
1 \\
15\end{array}$ & $\begin{array}{r}1 \\
5 \\
3 \\
3 \\
40\end{array}$ & $\begin{array}{l}2710.76 \\
2710.54 \\
2708.81 \\
2707.89 \\
2707.34\end{array}$ & $\begin{array}{r}5 \\
3 \\
10 \\
30 \\
2\end{array}$ & $\begin{array}{r}15 \\
8 \\
5 \\
100 \\
3\end{array}$ & $\begin{array}{l}2668.95 \\
2668.30 \\
2667.80 \\
2667.18 \\
2665.76\end{array}$ & $\begin{array}{l}5 d \\
1 \\
1 \\
8\end{array}$ & $\begin{array}{c}15 d \\
1 \\
2 \\
2 \\
20\end{array}$ & $\begin{array}{l}2626.66 \\
2626.42 \\
2625.99 \\
2625.51 \\
2624.38\end{array}$ & $\begin{array}{r}1 \\
5 \\
20 \\
2\end{array}$ & $\begin{array}{r}1 \\
3 \\
15 \\
60 \\
6\end{array}$ \\
\hline $\begin{array}{l}2749.30 \\
2749.21 \\
2747.40 \\
2744.35 \\
2743.84\end{array}$ & $\begin{array}{r}3 \\
3 \\
2 \\
\\
10\end{array}$ & $\begin{array}{r}8 \\
8 \\
5 \\
1 \\
30\end{array}$ & $\begin{array}{l}2706.94 \\
2702.96 \\
2702.47 \\
2702.30 \\
2701.65\end{array}$ & $\begin{array}{r}1 \\
30 \\
2 \\
2 \\
2\end{array}$ & $\begin{array}{r}3 \\
100 \\
5 \\
1 \\
4\end{array}$ & $\begin{array}{l}2665.10 \\
2664.69 \\
2663.95 \\
2663.17 \\
2662.66\end{array}$ & $\begin{array}{l}2 \\
4 h \\
2 \\
7\end{array}$ & $\begin{array}{c}6 \\
10 h \\
4 \\
1 \\
20\end{array}$ & $\begin{array}{l}2622.83 \\
2620.80 \\
2620.30 \\
2619.94 \\
2619.30\end{array}$ & $\begin{array}{l}2 \\
2 \\
1 \\
3 \\
3\end{array}$ & $\begin{array}{r}4 \\
5 \\
2 \\
10 \\
10\end{array}$ \\
\hline $\begin{array}{l}2742.76 \\
2742.54 \\
2741.17 \\
2740.02 \\
2739.28\end{array}$ & $\begin{array}{l}1 \\
\\
3 \\
3 h \\
1\end{array}$ & $\begin{array}{l}2 \\
2 h \\
8 \\
10 h l \\
2\end{array}$ & $\begin{array}{l}2700.67 \\
2699.80 \\
2699.42 \\
2697.60 \\
2697.39\end{array}$ & $\begin{array}{l}2 \\
2 \\
1 \\
2\end{array}$ & $\begin{array}{l}4 \\
6 \\
1 \\
2 \\
5\end{array}$ & $\begin{array}{l}2662.32 \\
2661.66 \\
2661.17 \\
2660.91 \\
2658.59\end{array}$ & $\begin{array}{r}5 \\
10 \\
1 \\
15\end{array}$ & $\begin{array}{r}3 \\
30 \\
3 \\
30 \\
2\end{array}$ & $\begin{array}{l}2618.77 \\
2617.58 \\
2616.07 \\
2615.88 \\
2615.15\end{array}$ & $\begin{array}{r}1 \\
2 \\
7 \\
30 \\
1\end{array}$ & $\begin{array}{l}2 \\
6 \mathrm{Fe} ? \\
20 \\
15 \\
3\end{array}$ \\
\hline $\begin{array}{l}2738.83 \\
2737.99 \\
2737.69 \\
2737.14 \\
2736.85\end{array}$ & $\begin{array}{r}40 \\
4 \\
4 \\
2 \\
30\end{array}$ & $\begin{array}{r}150 \\
2 \\
10 \\
6 \\
100\end{array}$ & $\begin{array}{l}2696.56 \\
2694.79 \\
2694.56 \\
2693.76 \\
2693.13\end{array}$ & $\begin{array}{r}15 \\
2 \\
4 \\
4\end{array}$ & $\begin{array}{r}30 \\
5 \\
1 \\
10 \\
2\end{array}$ & $\begin{array}{l}2658.19 \\
2657.98 \\
2657.59 \\
2657.34 \\
2657.04\end{array}$ & 2 & $\begin{array}{l}2 \\
1 \\
1 \\
1 \\
1\end{array}$ & $\begin{array}{l}2614.63 \\
2614.24 \\
2613.28 \\
2610.90 \\
2610.66\end{array}$ & $\begin{array}{r}1 \\
50 \\
3 \\
1\end{array}$ & $\begin{array}{r}3 \\
30 \\
10 \\
3 \\
2\end{array}$ \\
\hline $\begin{array}{l}2736.52 \\
2736.23 \\
2735.94 \\
2735.16 \\
2733.80\end{array}$ & $\begin{array}{l}3 \\
8 \\
2 \\
3 \\
1\end{array}$ & $\begin{array}{r}10 \\
7 \\
4 \\
8 \\
2\end{array}$ & $\begin{array}{l}2691.82 \\
2691.32 \\
2690.69 \\
2690.46 \\
2688.27\end{array}$ & $\begin{array}{l}8 \\
6 \\
1 \\
1 \\
6\end{array}$ & $\begin{array}{r}30 \\
20 \\
\\
2 \\
15\end{array}$ & $\begin{array}{l}2654.90 \\
2654.32 \\
2653.75 \\
2653.58 \\
2653.39\end{array}$ & $\begin{array}{l}1 \\
7 \\
2 \\
2\end{array}$ & $\begin{array}{l}1 \\
3 \\
6 \\
1 \\
1\end{array}$ & $\begin{array}{l}2610.00 \\
2609.04 \\
2608.88 \\
2608.15 \\
2607.26\end{array}$ & $\begin{array}{r}400 \\
\\
30 \\
3 \\
3\end{array}$ & $\begin{array}{r}800 \\
1 \\
10 \\
9 \\
9\end{array}$ \\
\hline $\begin{array}{l}2733.24 \\
2732.89 \\
2730.53 \\
2730.19 \\
2729.73\end{array}$ & $\begin{array}{c}1 \\
10 \\
20 \\
1 \\
1 h\end{array}$ & $\begin{array}{c}2 \\
5 \\
10 \\
2 \\
2 h\end{array}$ & $\begin{array}{l}2688.07 \\
2687.34 \\
2686.04 \\
2685.41 \\
2684.97\end{array}$ & $\begin{array}{c}1 \\
2 \\
1 \\
10 c\end{array}$ & $\begin{array}{c}2 \\
7 \\
3 \\
30 c \\
1\end{array}$ & $\begin{array}{l}2652.90 \\
2652.36 \\
2652.10 \\
2651.81 \\
2650.60\end{array}$ & $\begin{array}{r}3 \\
50 \\
1 \\
5+g ?\end{array}$ & $\begin{array}{r}8 \\
150 \\
1 \\
1 \\
10\end{array}$ & $\begin{array}{l}2605.83 \\
2605.41 \\
2604.88 \\
2603.74 \\
2602.55\end{array}$ & $\begin{array}{r}1 \\
10 \\
5 \\
2 \\
3\end{array}$ & $\begin{array}{r}2 \\
30 \\
15 \\
6 \\
7\end{array}$ \\
\hline $\begin{array}{l}2728.48 \\
2728.33 \\
2727.62 \\
2727.08 \\
2726.70\end{array}$ & $\begin{array}{r}4 \\
\\
2 \\
3 \\
30\end{array}$ & $\begin{array}{r}2 \\
1 \\
6 \\
8 \\
15\end{array}$ & $\begin{array}{l}2684.51 \\
2684.22 \\
2683.90 \\
2683.73 \\
2683.14\end{array}$ & $\begin{array}{l}5 \\
1 \\
3 \\
1 \\
5\end{array}$ & $\begin{array}{r}15 \\
2 \\
1 \\
2 \\
4\end{array}$ & $\begin{array}{l}2650.42 \\
2650.21 \\
2649.19 \\
2648.32 \\
2647.02\end{array}$ & $\begin{array}{r}1 \\
10 \\
20 \\
300\end{array}$ & $\begin{array}{r}1 \\
20 \\
60 \\
600\end{array}$ & $\begin{array}{l}2602.11 \\
2600.92 \\
2599.41 \\
2598.63 \\
2597.21\end{array}$ & $\begin{array}{r}10 \\
1 \\
1 \\
2 \\
15\end{array}$ & $\begin{array}{r}20 \\
3 \\
3 \\
5 \\
30\end{array}$ \\
\hline $\begin{array}{l}2726.18 \\
2725.66 \\
2724.20 \\
2723.75 \\
2723.40\end{array}$ & $\begin{array}{r}2 \\
15 \\
10 \\
2 \\
5\end{array}$ & $\begin{array}{r}4 \\
40 \\
30 \\
6 \\
15\end{array}$ & $\begin{array}{l}\text { 2682. } 77 \\
\text { 2682. } 67 \\
\text { 2682. } 33 \\
\text { 2681. } 60 \\
\text { 2681. } 21\end{array}$ & $\begin{array}{r}10 \\
10 \\
3 \\
1 \\
30\end{array}$ & $\begin{array}{r}20 \\
20 \\
8 \\
2 \\
100\end{array}$ & $\begin{array}{l}2646.26 \\
2645.60 \\
2645.38 \\
2644.51 \\
2643.02\end{array}$ & $\begin{array}{c}10 \\
2 \\
2 d \\
40 \\
15\end{array}$ & $\begin{array}{r}20 \\
4 \\
2 \\
150 \\
40\end{array}$ & $\begin{array}{l}2596.78 \\
2595.75 \\
2593.68 \\
2593.61 \\
2593.26\end{array}$ & $\begin{array}{l}5 \\
2 \\
2 \\
2 \\
2\end{array}$ & $\begin{array}{r}10 \\
1 \\
1 \\
5 \\
1\end{array}$ \\
\hline $\begin{array}{l}2723.02 \\
2721.56 \\
2719.80 \\
2719.31 \\
2718.19\end{array}$ & $\begin{array}{r}1 \\
10 \\
1\end{array}$ & $\begin{array}{r}1 \\
1 \\
3 \\
20 \\
2\end{array}$ & $\begin{array}{l}2680.96 \\
2680.67 \\
2679.43 \\
2679.03 \\
2678.58\end{array}$ & $\begin{array}{l}2 \\
3 \\
1 \\
1 \\
5\end{array}$ & $\begin{array}{r}6 \\
8 \\
2 \\
3 \\
10\end{array}$ & $\begin{array}{l}2642.54 \\
2642.38 \\
2642.13 \\
2641.28 \\
2640.49\end{array}$ & $\begin{array}{l}1 \\
3 \\
1 \\
2 \\
1\end{array}$ & $\begin{array}{l}2 \\
1 \\
2 \\
1 \\
2\end{array}$ & $\begin{array}{l}2593.06 \\
2592.82 \\
2591.15 \\
2589.88 \\
2588.93\end{array}$ & $\begin{array}{l}6 \\
6 \\
1 \\
6 \\
3\end{array}$ & $\begin{array}{r}20 \\
4 \\
2 \\
3 \\
8\end{array}$ \\
\hline $\begin{array}{l}2716.69 \\
2716.55 \\
2715.84 \\
2715.47 \\
2715.21\end{array}$ & $\begin{array}{l}4 \\
4 \\
4 \\
3 \\
2\end{array}$ & $\begin{array}{r}10 \\
10 \\
10 \\
8 \\
1\end{array}$ & $\begin{array}{l}\text { 2675. } 64 \\
\text { 2675. } 24 \\
\text { 2674. } 61 \\
\text { 2673. } 85 \\
\text { 2673. } 43\end{array}$ & $\begin{array}{r}2 \\
20 \\
1 \\
\\
20\end{array}$ & $\begin{array}{r}7 \\
50 \\
2 \\
1 \\
50\end{array}$ & $\begin{array}{l}2640.17 \\
2639.33 \\
2639.07 \\
2638.80 \\
2636.35\end{array}$ & $\begin{array}{c}10 c \\
1 \\
2 \\
2 \\
6\end{array}$ & $\begin{array}{c}20 c \\
3 \\
4 \\
6 \\
6\end{array}$ & $\begin{array}{l}2587.77 \\
2586.89 \\
2586.71 \\
2586.27 \\
2585.91\end{array}$ & $\begin{array}{l}1 \\
1 h \\
2 \\
3\end{array}$ & $\begin{array}{l}4 \\
4 h \\
3 \\
1 \\
4 \mathrm{Fe} ?\end{array}$ \\
\hline
\end{tabular}


TABLE 1. Arc and spark spectra of technetium-Continued

\begin{tabular}{|c|c|c|c|c|c|c|c|c|c|c|c|}
\hline \multirow{2}{*}{$\begin{array}{l}\lambda \\
A\end{array}$} & \multicolumn{2}{|c|}{ Intensity } & \multirow{2}{*}{$\begin{array}{l}\lambda \\
A\end{array}$} & \multicolumn{2}{|c|}{ Intensity } & \multirow{2}{*}{$\begin{array}{l}\lambda \\
A \\
A\end{array}$} & \multicolumn{2}{|c|}{ Intensity } & \multirow{2}{*}{$\begin{array}{l}\lambda \\
A\end{array}$} & \multicolumn{2}{|c|}{ Intensity } \\
\hline & Arc & Spark & & Arc & Spark & & Arc & Spark & & Arc & Spark \\
\hline $\begin{array}{l}2585.49 \\
2585.06 \\
2584.59 \\
2583.09 \\
2582.22\end{array}$ & $\begin{array}{l}1 d \\
1 h \\
3 h\end{array}$ & $\begin{array}{c}1 h \\
4 d \\
2 h \\
2 \\
10 h l\end{array}$ & $\begin{array}{l}2550.05 \\
2549.74 \\
2549.35 \\
2547.94 \\
2547.01\end{array}$ & $\begin{array}{r}20 \\
1\end{array}$ & $\begin{array}{r}10 \\
1 \\
1 \\
50 \\
4\end{array}$ & $\begin{array}{l}2503.56 \\
2503.29 \\
2502.36 \\
2501.86 \\
2501.38\end{array}$ & $\begin{array}{l}1 \\
1 \\
2\end{array}$ & $\begin{array}{l}4 \\
1 \\
1 \\
2\end{array}$ & $\begin{array}{l}2461.86 \\
2461.67 \\
2461.52 \\
2459.67 \\
2458.99\end{array}$ & $\begin{array}{l}1 \\
1 \\
2 \\
1 \\
2\end{array}$ & 4 \\
\hline $\begin{array}{l}2581.00 \\
2580.86 \\
2578.78 \\
2578.31 \\
2577.87\end{array}$ & $\begin{array}{l}3 \\
2 \\
15 h \\
15 \\
10\end{array}$ & $\begin{array}{l}7 \\
4 \\
40 h l \\
40 \\
20\end{array}$ & $\begin{array}{l}2545.75 \\
2545.55 \\
2544.42 \\
2543.24 \\
2541.38\end{array}$ & $\begin{array}{r}1 \\
3 \\
4 \\
500 \\
2\end{array}$ & $\begin{array}{c}2 \\
2 \\
8 \\
1000 \\
6\end{array}$ & $\begin{array}{l}2501.00 \\
2500.65 \\
2500.10 \\
2499.60 \\
2497.24\end{array}$ & $\begin{array}{r}3 \\
1 \\
1 \\
10\end{array}$ & $\begin{array}{r}1 \\
1 \\
1 \\
4 \\
20\end{array}$ & $\begin{array}{l}2456.47 \\
2456.17 \\
2455.95 \\
2454.48 \\
2453.97\end{array}$ & $2 d$ & $\begin{array}{l}1 \\
3 h \\
1 \\
3\end{array}$ \\
\hline $\begin{array}{l}2577.02 \\
2576.30 \\
2576.12 \\
2575.59 \\
2575.24\end{array}$ & $\begin{array}{r}1 \\
10 \\
1 \\
2 \\
5\end{array}$ & $\begin{array}{r}2 \\
30 \\
\\
1 \\
10\end{array}$ & $\begin{array}{l}2540.60 \\
2540.46 \\
2538.99 \\
2538.70 \\
2538.03\end{array}$ & $\begin{array}{l}2 \\
1 h \\
2 \\
1 \\
1 h\end{array}$ & $\begin{array}{l}5 \\
3 h \\
1 \\
4 \\
2 h\end{array}$ & $\begin{array}{l}\text { 2496. } 77 \\
2495.04 \\
\text { 2494. } 14 \\
\text { 2493. } 44 \\
\text { 2492. } 73\end{array}$ & $\begin{array}{r}100 \\
2 \\
4 \\
6\end{array}$ & $\begin{array}{r}200 \\
1 \\
1 \\
5 \\
3\end{array}$ & $\begin{array}{l}2453.70 \\
2452.42 \\
2451.76 \\
2450.63 \\
2450.10\end{array}$ & $\begin{array}{l}2 \\
3 \\
1 \\
2 \\
2\end{array}$ & $\begin{array}{l}1 \\
7 \\
3 \\
4 \\
4\end{array}$ \\
\hline $\begin{array}{l}2575.08 \\
2574.45 \\
2573.88 \\
2573.49 \\
2573.38\end{array}$ & $\begin{array}{l}5 \\
6 \\
1 h \\
1 \\
1\end{array}$ & $\begin{array}{c}10 \\
10 \\
2 h \\
2 \\
2\end{array}$ & $\begin{array}{l}2537.52 \\
2537.39 \\
2534.59 \\
2533.48 \\
2532.97\end{array}$ & $\begin{array}{l}1 \\
1 \\
5 \\
2 \\
4\end{array}$ & $\begin{array}{r}2 \\
2 \\
15 \\
1 \\
10\end{array}$ & $\begin{array}{l}\text { 2491. } 26 \\
2491.06 \\
2490.85 \\
2490.71 \\
2490.11\end{array}$ & $\begin{array}{l}2 \\
3 \\
1\end{array}$ & $\begin{array}{l}1 \\
6 \\
\\
2 h \\
3 h\end{array}$ & $\begin{array}{l}2449.12 \\
2448.62 \\
2447.89 \\
2447.45 \\
2446.86\end{array}$ & $\begin{array}{l}2 \\
1 \\
4 \\
1 \\
1\end{array}$ & $\begin{array}{l}6 \\
4 \\
7 \\
3\end{array}$ \\
\hline $\begin{array}{l}2573.17 \\
2572.92 \\
2571.57 \\
2571.18 \\
2569.89\end{array}$ & $\begin{array}{l}1 \\
1 \\
2 \\
2 \\
5\end{array}$ & $\begin{array}{r}2 \\
2 \\
3 \\
4 \\
15\end{array}$ & $\begin{array}{l}2532.04 \\
2531.38 \\
2530.35 \\
2528.10 \\
2527.63\end{array}$ & $\begin{array}{r}2 \\
3 \\
15 \\
1 \\
3\end{array}$ & $\begin{array}{r}8 \\
10 \\
40 \\
2 \\
10\end{array}$ & $\begin{array}{l}2489.47 \\
2489.06 \\
2488.03 \\
2487.98 \\
2487.74\end{array}$ & $\begin{array}{l}4 \\
2 \\
2\end{array}$ & $\begin{array}{l}6 \\
3 \\
3 h \\
3 \\
1\end{array}$ & $\begin{array}{l}2445.78 \\
2445.57 \\
2444.01 \\
2442.52 \\
2441.51\end{array}$ & $\begin{array}{l}1 \\
1\end{array}$ & $\begin{array}{l}3 \\
2 h \\
1 \\
1\end{array}$ \\
\hline $\begin{array}{l}2569.64 \\
2568.67 \\
2568.39 \\
2567.74 \\
2567.52\end{array}$ & $\begin{array}{l}1 \\
1 \\
3 d \\
1\end{array}$ & $\begin{array}{l}1 \\
2 \\
2 \\
8 d \\
2\end{array}$ & $\begin{array}{l}2525.77 \\
2525.27 \\
2525.05 \\
2523.88 \\
2523.41\end{array}$ & $\begin{array}{l}1 \\
1 \\
2 \\
5 c\end{array}$ & $\begin{array}{c}1 \\
3 \\
\\
5 \\
15 c\end{array}$ & $\begin{array}{l}2487.24 \\
2487.04 \\
2486.48 \\
2485.59 \\
2484.58\end{array}$ & $\begin{array}{l}2 \\
2 \\
2 \\
3 \\
2\end{array}$ & $\begin{array}{l}5 \\
3 \\
1 \\
8 \\
5\end{array}$ & $\begin{array}{l}2441.32 \\
2439.09 \\
2438.76 \\
2437.00 \\
2436.81\end{array}$ & $\begin{array}{l}1 \\
1 \\
3\end{array}$ & $\begin{array}{l}1 \\
\\
3 \\
2 \\
2\end{array}$ \\
\hline $\begin{array}{l}2567.04 \\
2566.33 \\
2566.03 \\
2565.67 \\
2565.17\end{array}$ & $\begin{array}{l}30 \\
2 h \\
3 \\
1\end{array}$ & $\begin{array}{c}100 \\
5 h \\
10 \\
1 \\
3\end{array}$ & $\begin{array}{l}2522.50 \\
2521.86 \\
2521.64 \\
2521.07 \\
2520.65\end{array}$ & $\begin{array}{l}4 d \\
2\end{array}$ & $\begin{array}{c}10 d \\
3 \\
1 \\
1 \\
3 h\end{array}$ & $\begin{array}{l}2482.60 \\
2481.17 \\
2481.00 \\
2480.70 \\
2479.24\end{array}$ & 5 & $\begin{array}{l}1 \\
1 \\
1 \\
2 \\
2\end{array}$ & $\begin{array}{l}2435.84 \\
2435.63 \\
2434.85 \\
2434.38 \\
2433.75\end{array}$ & 4 & $\begin{array}{l}2 \\
2 \\
1 \\
2\end{array}$ \\
\hline $\begin{array}{l}2564.67 \\
2563.32 \\
2563.12 \\
2562.82 \\
2562.55\end{array}$ & $\begin{array}{l}3 \\
1 \\
1 \\
2 \\
1\end{array}$ & $\begin{array}{l}5 \\
1 \\
1 \\
2\end{array}$ & $\begin{array}{l}2520.04 \\
2519.83 \\
2519.42 \\
2519.17 \\
2517.15\end{array}$ & $\begin{array}{c}2 \\
1 \\
1 \\
10+\mathrm{Si} \\
5\end{array}$ & $\begin{array}{l}4 \\
2 \\
3 \\
15 d \\
20\end{array}$ & $\begin{array}{l}2478.83 \\
2477.80 \\
2477.52 \\
2476.90 \\
2476.29\end{array}$ & 1 & $\begin{array}{l}2 \\
2 \\
1 \\
1 \\
5\end{array}$ & $\begin{array}{l}2432.62 \\
2431.39 \\
2431.11 \\
2429.55 \\
2423.25\end{array}$ & $\begin{array}{r}1 \\
15\end{array}$ & $\begin{array}{l}2 \\
1 \\
1 \\
2 \\
7\end{array}$ \\
\hline $\begin{array}{l}2562.01 \\
2561.15 \\
2560.63 \\
2560.17 \\
2559.43\end{array}$ & $\begin{array}{l}2 \\
1 \\
4 \\
.\end{array}$ & $\begin{array}{l}6 \\
3 \\
2 \\
1 \\
1\end{array}$ & $\begin{array}{l}2516.85 \\
2516.26 \\
2515.92 \\
2513.60 \\
2512.96\end{array}$ & $\begin{array}{l}5 \\
2 \\
1 \\
1\end{array}$ & $\begin{array}{c}15 h \\
4 \\
3 \\
\\
1\end{array}$ & $\begin{array}{l}2475.15 \\
2474.11 \\
2473.15 \\
2472.13 \\
2471.29\end{array}$ & $\begin{array}{l}3 \\
3\end{array}$ & $\begin{array}{r}5 \\
10 \\
2 \\
2 \\
3\end{array}$ & $\begin{array}{l}2421.29 \\
2420.79 \\
2418.69 \\
2418.05 \\
2417.82\end{array}$ & $\begin{array}{l}1 \\
1 \\
1 \\
2\end{array}$ & $\begin{array}{l}3 \\
3 \\
2 h \\
1 \\
1\end{array}$ \\
\hline $\begin{array}{l}2559.21 \\
2559.00 \\
2558.62 \\
2558.22 \\
2557.40\end{array}$ & $\begin{array}{r}1 \\
1 \\
40 \\
10 \\
2\end{array}$ & $\begin{array}{c}3 \\
150 \\
30 \\
5 h\end{array}$ & $\begin{array}{l}2512.27 \\
2511.28 \\
2510.17 \\
2509.45 \\
2508.83\end{array}$ & $\begin{array}{l}1 \\
1 \\
5 \\
2\end{array}$ & $\begin{array}{l}2 \\
3 \\
3 \\
1 \\
2\end{array}$ & $\begin{array}{l}2467.53 \\
2467.07 \\
2466.88 \\
2465.75 \\
2465.10\end{array}$ & $\begin{array}{l}1 h \\
4 \\
2 \\
5\end{array}$ & $\begin{array}{l}4 h \\
2 \\
2 \\
1 \\
3\end{array}$ & $\begin{array}{l}2417.53 \\
2417.10 \\
2416.70 \\
2416.23 \\
2414.47\end{array}$ & $\begin{array}{l}4 \\
2\end{array}$ & $\begin{array}{l}4 \\
1 \\
1 \\
2 \\
6\end{array}$ \\
\hline $\begin{array}{l}2556.84 \\
2556.10 \\
2554.78 \\
2554.36 \\
2550.78\end{array}$ & $\begin{array}{l}1 \\
3 \\
1 \\
1 h\end{array}$ & $\begin{array}{l}2 d \\
8 \\
3 \\
2 \\
1\end{array}$ & $\begin{array}{l}2508.39 \\
2507.99 \\
2507.14 \\
2505.04 \\
2503.98\end{array}$ & $\begin{array}{c}3 h \\
15 \\
2 \\
5\end{array}$ & $\begin{array}{r}1 \\
8 h \\
30 \\
7 \\
20\end{array}$ & $\begin{array}{l}2464.82 \\
2464.48 \\
2463.70 \\
2463.53 \\
2462.11\end{array}$ & $\begin{array}{r}3 \\
15 \\
1\end{array}$ & $\begin{array}{l}1 \\
7 \\
8 \\
2 h \\
2\end{array}$ & $\begin{array}{l}2412.62 \\
2411.30 \\
2410.20 \\
2408.82 \\
2408.16\end{array}$ & $\begin{array}{l}1 \\
1 \\
1 \\
2\end{array}$ & $\begin{array}{l}3 \\
1 \\
6\end{array}$ \\
\hline
\end{tabular}


TABLE 1. Arc and spark spectra of technetium-Continued

\begin{tabular}{|c|c|c|c|c|c|c|c|c|c|c|c|}
\hline \multirow{2}{*}{$\frac{\lambda}{A}$} & \multicolumn{2}{|c|}{ Intensity } & \multirow{2}{*}{$\begin{array}{l}\lambda \\
A\end{array}$} & \multicolumn{2}{|c|}{ Intensity } & \multirow{2}{*}{$\begin{array}{l}\lambda \\
A\end{array}$} & \multicolumn{2}{|c|}{ Intensity } & \multirow{2}{*}{$\begin{array}{l}\lambda \\
A\end{array}$} & \multicolumn{2}{|c|}{ Intensity } \\
\hline & Arc & Spark & & Arc & Spark & & Arc & Spark & & Arc & Spark \\
\hline $\begin{array}{l}2407.87 \\
2407.11 \\
2405.13 \\
2404.10 \\
2400.90\end{array}$ & $\begin{array}{l}1 \\
1 \\
1 \\
5 \\
1\end{array}$ & $\begin{array}{r}3 \\
15 \\
3\end{array}$ & $\begin{array}{l}2384.66 \\
2382.98 \\
2382.39 \\
2382.05 \\
2376.05\end{array}$ & $\begin{array}{l}4 \\
1 \\
6 \\
7\end{array}$ & $\begin{array}{r}10 \\
2 \\
3 \\
15\end{array}$ & $\begin{array}{l}2360.44 \\
2354.08 \\
2353.16 \\
2349.17 \\
2346.59\end{array}$ & & $\begin{array}{l}1 \\
1 \\
1 \\
1 \\
2\end{array}$ & $\begin{array}{l}\text { 2316. } 72 \\
2315.32 \\
2313.91 \\
2300.97 \\
2300.44\end{array}$ & & $\begin{array}{l}1 \\
2 \\
1 \\
1 \\
1\end{array}$ \\
\hline $\begin{array}{l}2398.67 \\
2396.64 \\
2394.97 \\
2394.49 \\
2393.42\end{array}$ & $\begin{array}{l}7 \\
2\end{array}$ & $\begin{array}{r}20 \\
1 \\
1 \\
1 \\
1\end{array}$ & $\begin{array}{ll}2373 . & 77 \\
2369.05 \\
2368.35 \\
2368.01 \\
2367.67\end{array}$ & & $\begin{array}{l}1 \\
2 \\
1 \\
1 \\
1\end{array}$ & $\begin{array}{l}2345.17 \\
2344.84 \\
2344.71 \\
2341.67 \\
2331.78\end{array}$ & $\begin{array}{l}1 \\
1\end{array}$ & $\begin{array}{l}3 \\
3 \\
1 \\
2 h \\
2\end{array}$ & $\begin{array}{l}\text { 2299. } 96 \\
2298.14 \\
2292.14 \\
2291.64 \\
2285.47\end{array}$ & 1 & $\begin{array}{r}1 \\
10 \\
1 \\
1 \\
3\end{array}$ \\
\hline $\begin{array}{l}2392.02 \\
2390.93 \\
2389.36 \\
2386.03 \\
2385.32\end{array}$ & 2 & $\begin{array}{l}1 \\
1 \\
1 \\
1 \\
1\end{array}$ & $\begin{array}{l}\text { 2366. } 55 \\
2365.91 \\
2365.53 \\
2361.80 \\
2360.78\end{array}$ & 2 & $\begin{array}{l}4 \\
2 \\
1 \\
2 \\
2\end{array}$ & $\begin{array}{l}2329.49 \\
2328.41 \\
2327.69 \\
2324.57 \\
2318.74\end{array}$ & $\begin{array}{l}1 \\
1\end{array}$ & $\begin{array}{l}1 \\
3 \\
1 \\
1\end{array}$ & $\begin{array}{l}2282.41 \\
2280.33 \\
2272.45 \\
2271.14 \\
2266.24 \\
2261.30\end{array}$ & $1 d$ & $\begin{array}{l}1 \\
2 \\
1 \\
4 d \\
2 \\
1\end{array}$ \\
\hline
\end{tabular}

\section{Results}

The results of this investigation are presented in table 1, where wavelengths and relative intensities of 2,121 spectral lines characteristic of technetium atoms and ions are given. In this table the wavelengths represent the averages of two to six independent determinations. Several hundred faint lines that were measured only once on the strongest spectrograms have been omitted from table 1 , but some of these may be reported later if any confirm predictions from atomic energy levels.

The average probable error in these wavelengths is believed to be less than $\pm 0.01 \mathrm{~A}$. There are three reasons for this belief, namely, computed probable errors of lines measured on six spectrograms, agreement of the wavelengths of impurity lines with their accepted values, and the average difference between the measured values of classified lines and their values computed from atomic energy levels derived from these observations [14].

In addition to six-figure wavelengths, estimated relative intensities of arc and spark lines of Tc are given in table 1 . These intensities range from 1,000 for the strongest line to 1 , which represents the weak lines whose reality is unequivocal because they were observed two or more times. In the spectral range in which arc and spark intensities were compared $(4700$ to 2200 A) Te I lines have nearly double intensity in the arc, whereas Te II lines have about treble intensity in the spark. Most of the Tc II and Te I line-intensities thus differ by a factor of 5 or 6 , and their assignment is unambiguous. A few lines, mostly faint ones, appear to have about the same intensity in arc and spark, but naturally a few approxmate coincidences of $\mathrm{Tc} \mathrm{I}$ and $\mathrm{T}$ c II lines may be expected.

Occasionally the intensity numbers are accompanied by literal symbols having the meanings given in the table. Some of these symbols are especially significant for Tc spectra because they suggest hyperfine structure due to interaction of valence electrons with atomic nuclei. This hyperfine structure was observed in manganese spectra and in rhenium spectra, unresolved by gratings in the former case but easily resolved in the latter. The hyperfine structure of Tc lines appears to be intermediate between Mn and Re. Many Tc lines, especially among longer waves, appear as flat-topped images, four or five times as wide as the slit. These lines are under investigation with interferometers.

Besides a general resemblance of Tc spectra with those of Mn and Re, we can now define in detail the analogous spectral lines of the three elements or their ions. For neutral a toms of Mn, Tc, or Re the resonance lines are known as $d^{5} s^{2}{ }^{6} \mathrm{~S}_{21 / 2}-d^{5} s^{1} p^{16} \mathrm{P}_{31 / 3},{ }_{21 / 3},{ }_{11 / 2}$, and the respective wavelengths are 4030.76 , 4033.07, 4034.49 A in Mn; 4297.06, 4262.26, 4238.19 $\mathrm{A}$ in Tc; and $3460.47,3464.72,3451.88 \mathrm{~A}$ in Re. For singly ionized atoms of $\mathrm{Mn}, \mathrm{Tc}$, or Re the resonance lines are known as $d^{5} s^{1}{ }^{7} \mathrm{~S}_{3}-d^{5} p^{1}{ }^{7} \mathrm{P}_{4,3,2}$, and the respective wavelengths are $2576.10,2593.73,2605.69 \mathrm{~A}$ for $\mathrm{Mn}^{+} ; 2543.24,2610.00,2647.02 \mathrm{~A}$ for $\mathrm{Tc}^{+}$; but not yet revealed for $\mathrm{Re}^{+}$. Full details concerning the interpretations of lines in the first two spectra of Tc will be divulged in another paper. The above-mentioned resonance lines of $\mathrm{Tc}$ and $\mathrm{Tc}^{+}$are probably useful for detecting this element in concentrations of $10^{-7}$; the fact that they cannot be found in any spectral tables excluding fission products may be regarded as proof that technetium is not spectroscopically detectable in nature.

The authors acknowledge the generous cooperation of the United States Atomic Energy Commission in lending samples of technetium for this investigation, and compliment G. W. Parker of the 
Oak Ridge National Laboratory on the high purity of these samples. Assistance with wavelength calculations was given by W. Lyle, R. Cross, and R. Murphy, and the direct-current arc spectrograms were made by W. Bozman.

\section{References}

[1] D. I. Mendeléeff, J. Russ. Chem. Soc. 1, 60 (1869).

[2] H. J. G. Moseley, Phil. Mag. 26, 1024 (1913); 27, 703 (1914).

[3] W. Noddack, I. Tacke, und O. Berg, Naturwissenschaften 13, 567 (1925)

[4] W. F. Meggers, J. Research NBS 6, 1027 (1931) RP322.

[5] E. Segré, Sci. Monthly 5\%, 12 (1943).
[6] C. Perrier and E. Segré, J. Chem. Phys. 5, 712 (1937); 7, 155 (1939).

[7] C. Perrier and E. Segré, Nature 159, 24 (1947).

[8] Plutonium Project, J. Am. Chem. Soc. 68, 2411 (1946).

[9] G. W. Parker, J. Reed, and J. W. Ruch, CNL-1, Clintor National Laboratory, Contract No. W-35-058-ing-71, (Jan. 9, 1948).

[10] M. G. Inghram, Phys. Rev. 72, 1269 (1947).

[11] D. L. Timma, J. Optical Soc. Am. 39, 898 (1949).

[12] L. E. Burkhart, W. F. Peed and B. G. Saunders, Phys. Rev. 73, 347 (1948).

[13] W. F. Meggers and B. F. Scribner, Y-476, Oak Ridge Spectroscopy Symposium, Abstracts of Papers (March 24 to 25,1949 ).

[14] W. F. Meggers and B. F. Scribner, J. Opt. Soc. Am. 39, 1059 (Abstract) (1949).

Washington, August 21, 1950.

\title{
Dissociation Constants of 4-Aminobenzophenone Calcu- lated From Ultraviolet Spectra at Several Temperatures
}

\author{
By Elizabeth E. Sager and Iris J. Siewers
}

\begin{abstract}
Many of the physical-chemical properties of compounds of low solubility are difficult to determine because of the necessarily small concentrations of material. In many cases advantage may be taken of measurements of the absorption spectra if the bands occur in favorable ranges of the spectrum. The diphenyl ketones fall in this category and have been the subject of recent studies.

The ultraviolet absorption spectra of several concentrations of benzophenone, 4-aminobenzophenone, and 4, $4^{\prime}$-diaminobenzophenone have been measured and their molar absorbencies determined. The dissociation of 4-aminobenzophenone, a weak base, has been carefully studied at temperatures from $10^{\circ}$ to $40^{\circ} \mathrm{C}$ in 5-degree steps. Dissociation constants for each temperature, as well as activity coefficient terms, have been calculated. The heat of dissociation has also been determined.
\end{abstract}

\section{Introduction}

The acidic or basic properties of many slightly soluble compounds have not been determined because of experimental difficulties in applying the usual titration or electromotive-force methods to very low concentrations. Diphenylketones, such as benzophenone, 4-aminobenzophenone and 4,4'diaminobenzophenone, are practically insoluble in water and fall in this category. They are colorless in aqueous solution, but their aromatic structure suggests that their ultraviolet spectra may be used to study some of their chemical properties. Accurate spectrophotometric measurements of concentrations as low as $10^{-4}$ or $10^{-5}$ molar can now be made at many temperatures. The observed changes in spectral absorbancy with changes in hydrogen-ion concentrations can be used to calculate dissociation constants and other related thermodynamic quantities.

The molar absorbancies of the molecular and ionic states of benzophenone, 4-aminobenzophenone, and $4,4^{\prime}$-diaminobenzophenone were first determined and compared. The parent compound, benzophenone, has no group to ionize, and its spectral curves are identical whether the material is in aqueous, acid, or basic medium. 4-Aminobenzophenone is a weak base and does not dissociate appreciably in aqueous solution, as demonstrated by identical curves of the free base in water and in various concentrations of alkali. Moderately strong acid is required to effect complete dissociation, as evidenced by the entirely different absorbancy curve in the near ultraviolet. $4,4^{\prime}$-Diaminobenzophenone has two groups whose dissociation ranges overlap, and extensive experimental data will be required to determine the constants.

A comprehensive study of the dissociation of 4aminobenzophenone has been made at several temperatures, and the constants at $10^{\circ}, 15^{\circ}, 20^{\circ}, 25^{\circ}$, $30^{\circ}, 35^{\circ}$, and $40^{\circ} \mathrm{C}$ have been calculated and are reported in this paper. Hydrochloric acid was used to control the hydrogen-ion concentrations at various stages during the dissociation. The contribution of the $10^{-5}-M$ base to ionic strengths of $10^{-3}$ and $10^{-2}-M$ hydrochloric acid is so small that it can be neglectedthus the hydrogen-ion concentration may be taken as that of the known amount of hydrochloric acid.

The limiting spectral absorbancy curves of the free base and of the ionized compound do not change with change in temperature in the range studied, which is very fortunate. At a given temperature, then, all changes in absorbancy for one dissociation series may be attributed solely to the dissociation reaction. Another favorable factor is that 4-aminobenzophenone dissociates over a $\mathrm{pH}$ range of 1 to 3 , where the temperature has little influence upon $\mathrm{pH}$ values. 